(Supporting Information)

\title{
Inducing Disorder in Order: Hierarchically Porous Covalent Organic Framework Nanostructures for Rapid Removal of Persistent Organic Pollutants
}

Suvendu Karak, ${ }^{1,2}$ Kaushik Dey, ${ }^{3}$ ArunTorris, ${ }^{4}$ Arjun Halder, ${ }^{1,2}$ Saibal Bera ${ }^{1,2}$ Fayis Kanheerampockil ${ }^{4}$ and Rahul Banerjee, ${ }^{3, *}$

${ }^{1}$ Academy of Scientific and Innovative Research, New Delhi, India.

${ }^{2}$ Physical /Materials Chemistry Division, CSIR-National Chemical Laboratory, Dr. Homi Bhabha Road, Pune-411008, India.

${ }^{3}$ Department of Chemical Sciences, Indian Institute of Science Education and Research, Kolkata, Mohanpur 741246, India.

${ }^{4}$ Polymers Science and Engineering Division, CSIR-National Chemical Laboratory, Dr. Homi Bhabha Road, Pune-411008, India.

*Email: r.banerjee@ncl.res.in; Tel: +91-20-2590-2535. 


\section{Table of Contents}

\begin{tabular}{|c|l|c|}
\hline Section & & Page No \\
\hline S-1 & Materials and Instrumentation & S3 \\
\hline S-2 & Synthetic procedures & S5 \\
\hline S-3 & Structure Refinement and Scherrer's Analysis & S8 \\
\hline S-4 & FTIR spectra & S12 \\
\hline S-5 & ${ }^{13}$ C CP-MAS spectra and TG analyses & S13 \\
\hline S-6 & SEM and TEM analyses & S14 \\
\hline S-7 & $\begin{array}{l}\text { Micro-scale X-ray Computed Tomography and mechanical } \\
\text { stability }\end{array}$ & S15 \\
\hline S-8 & Nitrogen adsorption analysis & S19 \\
\hline S-9 & Mechanism of Foam Synthesis & S21 \\
\hline S-10 & Adsorption of pollutants & S21 \\
\hline S-11 & Binding energy calculation & S36 \\
\hline S-12 & Numerical simulation studies & S37 \\
\hline S-13 & References & \\
\hline
\end{tabular}




\section{Section S-1: Materials and Instrumentation}

\section{Materials}

The starting materials 1,3,5-triformylphloroglucinol (Tp) was synthesized in the lab following the previous literature protocols. ${ }^{1,2}$ All commercially available reagents and solvents were used without further purification. All the commercially available materials were bought from Sigma-Aldrich, TCI chemicals, Avra Chemicals and Fisher Scientific depending upon their availability.

\section{General instrumentations and methods}

Powder X-ray diffraction (PXRD) data were collected using a Rigaku, MicroMax-007HF with high-intensity Microfocus rotating anode X-ray generator. All the COFs were recorded in the $2 \theta$ range between $2-40^{\circ}$ and data was collected with the help of Control Win software. The radiation used was $\mathrm{Cu} \mathrm{K} \alpha(\lambda=1.54 \AA)$ with a Ni filter, and the data collection was carried out using an Aluminium holder at a scan speed of $1^{\circ} \min ^{-1}$ and a step size of $0.02^{\circ}$.

Fourier transform infrared (FT-IR) spectra were obtained using a Bruker Optics ALPHAE spectrometer with a universal Zn-Se ATR (attenuated total reflection) accessory. FT-IR data are reported with a wave number $\left(\mathrm{cm}^{-1}\right)$ scale.

Thermogravimetric analyses (TGA) were carried out on a TG50 analyzer (Mettler-Toledo) and a SDT Q600 TG-DTA analyzer under $\mathrm{N}_{2}$ atmosphere at a heating rate of $10{ }^{\circ} \mathrm{C} \mathrm{min}{ }^{-1}$ within a temperature range of $30-900{ }^{\circ} \mathrm{C}$.

Scanning Electron Microscopy (SEM) measurements were executed with a Zeiss DSM 950 scanning electron microscope and FEI, QUANTA 200 3D Scanning Electron Microscope equipped with tungsten filament as electron source operated at $10 \mathrm{kV}$. The samples were prepared simply by putting a drop of COFs dispersed in isopropanol on a clean piece of Silicon wafer. To avoid charging during SEM analyses, we coated all the COFs samples with a thin layer of gold by a SCD 040 Balzers Union prior to analyses.

Transmission Electron Microscopy (TEM) analyses were performed using a FEI Tecnai G2 F20 X-TWIN TEM at an accelerating voltage of $200 \mathrm{kV}$. The TEM Samples were 
prepared for analyses by drop casting the samples (dispersed in isopropanol) on copper grids TEM Window (TED PELLA, INC. 200 mesh).

$\mathbf{N}_{2}$ adsorption analyses were performed at $77 \mathrm{~K}$ using a liquid nitrogen bath $(77 \mathrm{~K})$ on a Quantachrome Quadrasorb automatic volumetric instrument. All the COFs samples were outgassed for $12 \mathrm{~h}$ at $120{ }^{\circ} \mathrm{C}$ under vacuum prior to the gas adsorption studies. The surface areas were evaluated using Brunauer-Emmett-Teller (BET) model applied between $\mathrm{P} / \mathrm{P}_{0}$ values of 0.05 and 0.3 for mesoporous COFs. The pore size distributions were calculated using the non-localized density functional theory (NLDFT) method. The surface area of each of the COF has been measured multiple times and then averaged out for proper comparison.

Solid state NMR spectra were recorded (SSNMR) on Bruker 300 MHz NMR spectrometer. Carbon chemical shifts are expressed in parts per million ( $\delta$ scale).

Atomic Force Microscopy (AFM) imaging technique was used with AC 240 TS-R3 silicon cantilever probes. Topographic images, amplitude of the samples were obtained and analyzed by standard AC mode imaging. And force curve and mapping were taken by force measurement for nano mechanical mapping measurement (All the Nano mechanical measurements were calculated using Herts model).

Confocal laser scanning microscope (CLSM) (Zeiss, Oberkochen, Germany Model LSM 710) was used to achieve background free high resolution images of micro porous COFfoam. The confocal microscope was equipped with EC Epiplan-APOCHROMAT objective of 50X magnification and a numerical aperture of 0.9. Fluorescence from the sample was excited with the 488-nm line of an Argon laser. The fluorescence was separated from the excitation light by band pass sliders $(493 \mathrm{~nm}-619 \mathrm{~nm})$ in front of spectral detectors. The microscope objective was attached to an opto-electronically coded focus z-driver to scan the objective along the vertical $z$ direction and produce three dimensional images. A pinhole was used to reject the out-of-plane light for background rejection hence better signal to noise ratio. We typically acquired 150-200 sliced stacks of 512 X 512 pixel images in the horizontal $x-y$ plain separated by $0.25 \mathrm{um}$ in the $z$ direction. The length of the full-sized field of view is approximately $283 \mathrm{um}$ and a depth approximately $40 \mu \mathrm{m}$. The images were processed using the Zen software (Zeiss, Oberkochen). Images of each plain were converted to 2.5 dimensional (2.5D) images to understand the absorbed dye intensity profile on the 
surface and internal microspores (graphical illustration of intensities per pixel for each plain using grayscale).

UV-vis spectra were measured using Shimadzu UV-vis-IR-3600 plus spectrophotometer and Chemito, Spectrascan UV 2700 spectrophotometer.

\section{Section S-2: Synthetic Procedures and methods}

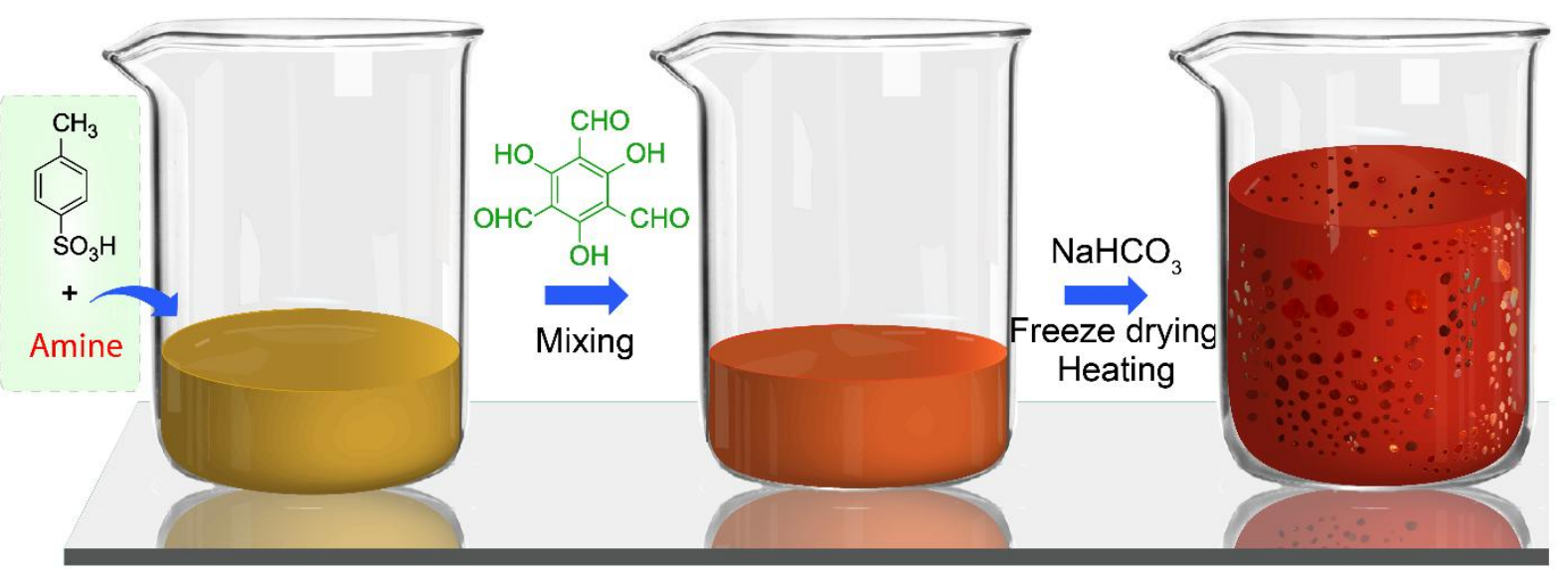

Figure S1 | Synthesis of COF-foam. COF-foams have been synthesized following the sequential steps. P-Toluene sulfonic acid and various diamines wer mixed and reacted with aldehyde followed by the addition of water. Sodium bicarbonate addition, lyophilization, and heating resulted hierarchically porous COF-foams.

Synthesis of COF-foams. 2 gm of $p$-toluene sulphonic acid (monohydrate) was taken in a mortar. Then $0.45 \mathrm{mmol}$ of corresponding diamine [ $p$-phenylenediamine (2,5-dimethyl- $p$ phenylenediamine $(\mathrm{Pa}-2,61.3 \mathrm{mg})$ for TpPa-2-foam; 2-nitro-1,4-phenelynediamine $\left(\mathrm{Pa}-\mathrm{NO}_{2}\right.$, $68.9 \mathrm{mg}$ ) for $\mathrm{TpPa}-\mathrm{NO}_{2}$-foam; 3,3'-dimethylbenzidine (BD-Me - -foam, $95.5 \mathrm{mg}$ ) for TpBD$\mathrm{Me}_{2}$-foam; $p$-Azoaniline (Azo, $95.5 \mathrm{mg}$ ) for TpAzo-foam] was added. The reaction mixture was grounded thoroughly with a pestle. Then there was the addition of $0.3 \mathrm{mmol}(63 \mathrm{mg})$ of 1,3,5-triformylphloroglucinol (Tp). The continuous mixing was performed for another few minutes until it was a uniform mixture. Then there was the addition of few drops of water to the mixture and then the mixture was ground again for another 10 to 15 minutes. The addition of water resulted in a paste like material. Then $500 \mathrm{mg}$ of sodium bicarbonate $\left(\mathrm{NaHCO}_{3}\right)$ was added to it in few batches. After each addition the paste was mixed thoroughly and few drops 
of water was added. This makes the paste very much fluffy in nature. Then the mixture was transferred into vial for fridge drying for 5 hrs. After complete removal of water, the vial was kept for heating in an oven at $90{ }^{\circ} \mathrm{C}$ for $12 \mathrm{hrs}$. Even during the heating, excess sodium bicarbonate emits carbon dioxide and that leaves the foam with heterogeneous pores. The resultant foam was washed several times with hot water in order to remove off the unreacted PTSA and excess bicarbonate. Monomers and oligomers were washed off with the sequential treatment of $N, N$-dimethylacetamide (DMAc), water and acetone. The foam was dried under vacuum with heating before further use.

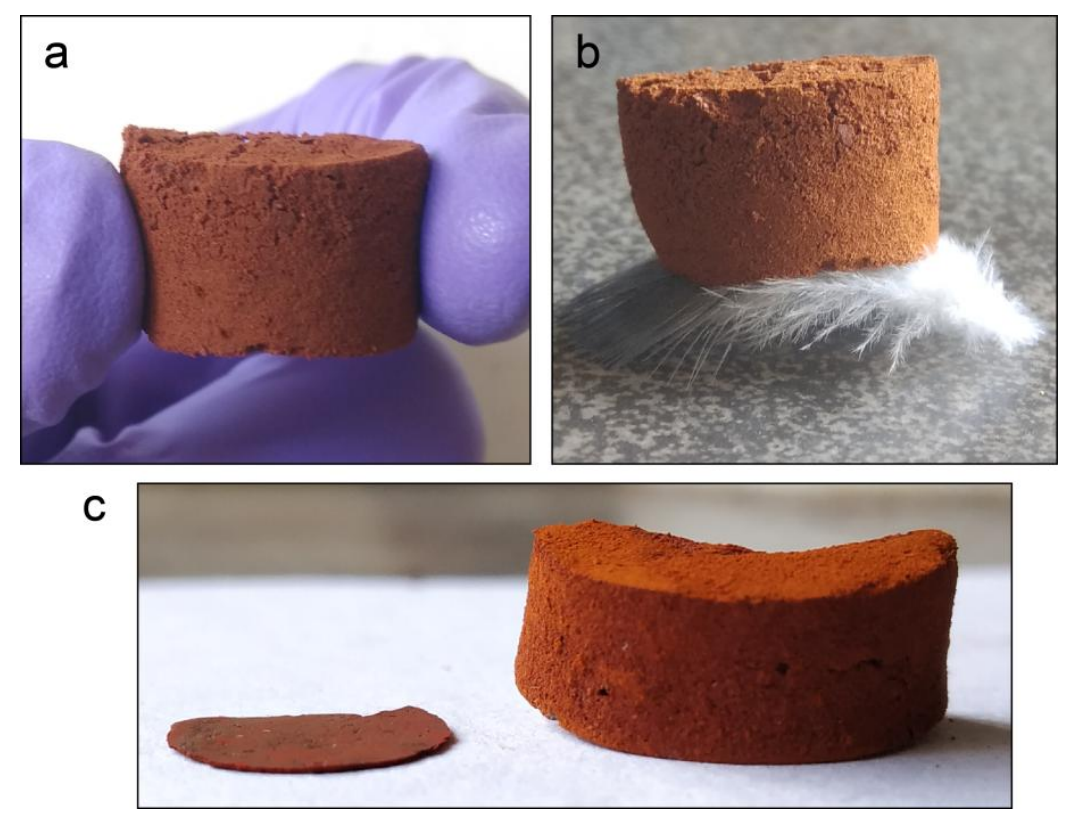

Figure S2 | Digital images of foam. The foam shows discrete physical structure a). They are also light weight $b$ ) and there is a certain volume expansion c) as compared to the COF. The volume of the COF-foam has been compared to that of the COF of same weight.

The highly crystalline and uniformly porous COFs were synthesized following the saltmediated crystallization approach. However, during the synthesis of foams, crystallization, as well as defect formation occur simultaneously in the presence of acid and sodium bicarbonate. The drop in the surface area of the COF-foams as compared to the corresponding COF could be attributed to the lower crystallinity (smaller crystallite size) due to the random defects inside the layered structure. The comparison of their properties has been mentioned here (Table S1) to evaluate the synthetic strategy. 
Table S1 | Comparison of properties between COF and corresponding foam.

\begin{tabular}{|c|c|c|c|c|c|c|c|c|}
\hline \multirow{2}{*}{ Properties } & \multicolumn{2}{|c|}{ TpPa-2 } & \multicolumn{2}{|c|}{$\mathrm{TpPa}-\mathrm{NO}_{2}$} & \multicolumn{2}{|c|}{ TpAzo } & \multicolumn{2}{|c|}{$\mathrm{TpBD}-\mathrm{Me}_{2}$} \\
\hline & $\mathrm{COF}$ & Foam & $\mathrm{COF}$ & Foam & $\mathrm{COF}$ & Foam & $\mathrm{COF}$ & Foam \\
\hline $\begin{array}{c}\text { Surface area } \\
\left(\mathrm{m}^{2} \mathrm{~g}^{-1}\right)\end{array}$ & 538 & 579 & 850 & 254 & 3038 & 1054 & 3109 & 797 \\
\hline Pore size $(\AA)$ & 10.6 & $\begin{array}{c}\text { No } \\
\text { uniform } \\
\text { pore size }\end{array}$ & 13.2 & $\begin{array}{c}\text { No } \\
\text { uniform } \\
\text { pore size }\end{array}$ & 25.8 & $\begin{array}{c}\text { No } \\
\text { uniform } \\
\text { pore size }\end{array}$ & 16.3 & $\begin{array}{c}\text { No } \\
\text { uniform } \\
\text { pore size }\end{array}$ \\
\hline
\end{tabular}

Adsorption studies. The highest removal efficiency was measured in a $15 \mathrm{~mL}$ culture vial with $5 \mathrm{mg}$ COF-foam followed by the addition of $5 \mathrm{~mL}$ stock pollutants. After continuous stirring for $2 \mathrm{hrs}$ at ambient temperature the solution was filtered with a syringe having 0.22 $\mu \mathrm{m}$ nylon membrane filter. The stock pollutant solution was also passed through the membrane for ready comparison of the removal efficiency before UV-vis spectroscopy measurement.

The pollutant removal efficiency was measured by the following equation.

$$
\text { Pollutant removal efficiency }(\%)=\frac{C_{0}-C_{t}}{C_{0}} \times 100
$$

where $C_{0}\left(\mathrm{mmol} \mathrm{l}^{-1}\right)$ and $C_{t}\left(\mathrm{mmol} \mathrm{l}^{-1}\right)$ are the initial and residual concentration (after adsorption) of pollutants in the stock solution and filtrate, respectively.

Kinetic adsorption studies were performed in $25 \mathrm{ml}$ beakers equipped with magnetic stir bars at ambient temperature with constant stirring rate of 450 r.p.m. Firstly, the foam was taken in $5 \mathrm{~mL}$ of water and then sonicated for 5 minutes. After that the COF-foam was kept for stirring for another 15 minutes on a stirrer for uniform mixture. Then, $10 \mathrm{~mL}$ pollutant stock was added to foam in order to get final pollutant concentration of $10 \mu \mathrm{M}$. The kinetic adsorption study was measured from the measurement of removal efficiency at different time interval. At any time $\mathrm{t}$, if $q_{t}$ is the amount of pollutant adsorbed per gm of COF-foam adsorbent then

$$
q_{t}=\frac{\left(C_{0}-C_{t}\right) M_{w}}{m}
$$

where $m$ is the mass ( $\mathrm{g}$ ) of sorbent (COF-foam) used in this study and $M_{w}$ is the molar mass $\left(\mathrm{g} \mathrm{mol}^{-1}\right)$ of the pollutant.

The rate of adsorption of pollutant was calculated following the Ho and McKay's pseudosecond-order adsorption model. The equation can be represented as: 


$$
\frac{t}{q_{t}}=\frac{t}{q_{e}}+\frac{1}{k_{o b s} q_{e}^{2}}
$$

where $q_{e}$ is the amount of pollutant adsorbed $\left(\mathrm{mg} \mathrm{g}^{-1}\right)$ at equilibrium time and $k_{o b s}$ is the pseudo second order rate constant $\left(\mathrm{g} \mathrm{mg}^{-1} \mathrm{~min}^{-1}\right)$ of pollutant adsorption.

In order to get the maximum uptake capacity of pollutants at equilibrium $\left(q_{\max , e}\right)$ of the COF-foam Langmuir model was followed as:

$$
\frac{1}{q_{e}}=\frac{1}{q_{\max , e}}+\frac{1}{q_{\max , e} K c}
$$

where $q_{e}\left(\mathrm{mg} \mathrm{g}^{-1}\right)$ is the amount of pollutant adsorbed at equilibrium time, $q_{\max , e}\left(\mathrm{mg} \mathrm{g}^{-1}\right)$ is the maximum adsorption capacity of COF-foam at equilibrium, $K\left(\mathrm{~mol}^{-1}\right)$ is the equilibrium constant and $c\left(\mathrm{~mol} \mathrm{l}^{-1}\right)$ is the residual pollutant concentration at equilibrium.

Flow-through adsorption studies. In order to check the continuous pollutant removal efficiency of the COF-foam, flow-through experiment was performed. $10 \mathrm{mg}$ COF-foam was taken in $10 \mathrm{~mL}$ water and then it was kept for sonication for 5 minutes. The uniform mixture was then stirred for 10 minutes at 450 r.p.m. in ambient temperature. Then the mixture was packed inside a column and the COF-foam was treated as column bed for pollutants. Then 10 $\mathrm{mL} 25 \mu M \mathrm{MB}$ solution was passed through the column bed in order to get the complete removal of pollutants. The flow rate was also controlled with compressed air which does not impact the performance of the COF-foam.

\section{Section S-3: Structure Refinement and Scherrer's Analysis}

However, as per reviewer's suggestion, the result has been further justified with Scherrer's analysis by achieving the domain size of the COF crystallites. The measurement has been done considering the Gaussian fitting of the high intense first peak (diffraction from $<100>$ plane). The analysis depicts that the COF crystallites size could vary when a particular COF has been synthesized from different salts (Table 1).

The Scherrer's equation can be written as: 


$$
\tau=\frac{K \lambda}{\beta \cos \theta}
$$

where:

$\tau$ is the size of the ordered (crystalline) domains;

$K$ is a dimensionless shape factor, with a value close to unity. The shape factor varies with the actual shape of the crystallite. Herein we have considered the shape factor as unity for the ease of calculation.

$\lambda$ is the X-ray wavelength which has the value $1.5418 \AA$;

$\beta$ is the line broadening at half of the maximum intensity (FWHM), after subtracting the instrumental line broadening, in radians. Silver behenate $\left(\mathrm{C}_{22} \mathrm{H}_{44} \mathrm{O}_{2} \cdot \mathrm{Ag}\right)$ was used to determine the instrumental peak broadening.

$$
\beta \exp ^{2}-\beta i n s^{2}=\beta^{2}
$$

Bins and $\beta$ exp are the line broadening of the standard sample and COF samples respectively.

$\theta$ is the Bragg angle (in degrees).

Table S2 | Domain size of the COF crystallites $(\AA)$ synthesized from different salts.

\begin{tabular}{|c|c|c|}
\hline Name & $\begin{array}{c}\text { COF-foam crystallite size } \\
(\AA)\end{array}$ & COF crystallite size $(\AA)$ \\
\hline TpPa-2 & 79.5 & 132 \\
\hline $\mathrm{TpPa}^{\mathrm{A}} \mathrm{NO}_{2}$ & 74.4 & 238.9 \\
\hline $\mathrm{TpBD}_{2} \mathrm{Me}_{2}$ & 30 & 399.4 \\
\hline TpAzo & 75.4 & \\
\hline
\end{tabular}




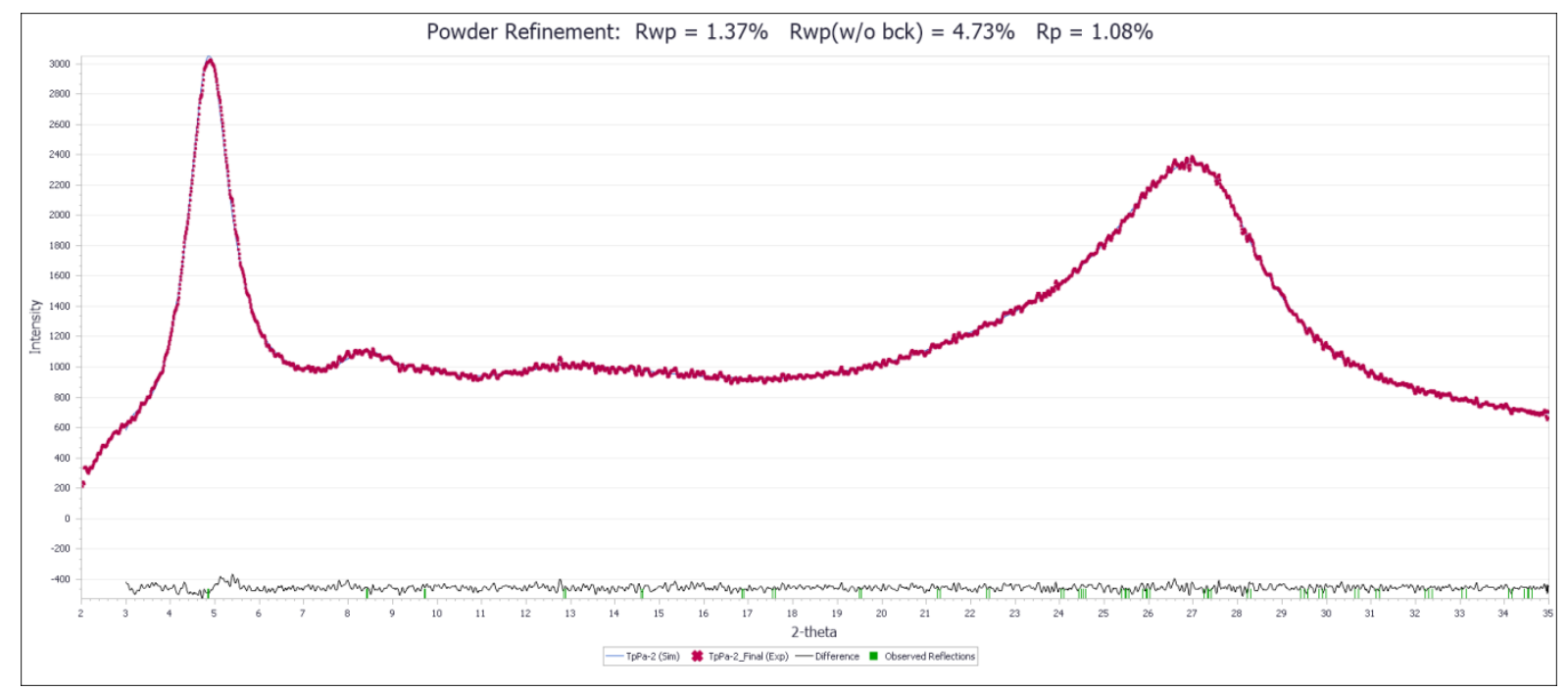

Figure S3 | Pawley refinement for TpPa-2-foam. Pawley refinement indicates the agreement between the simulated and experimental PXRD patterns.

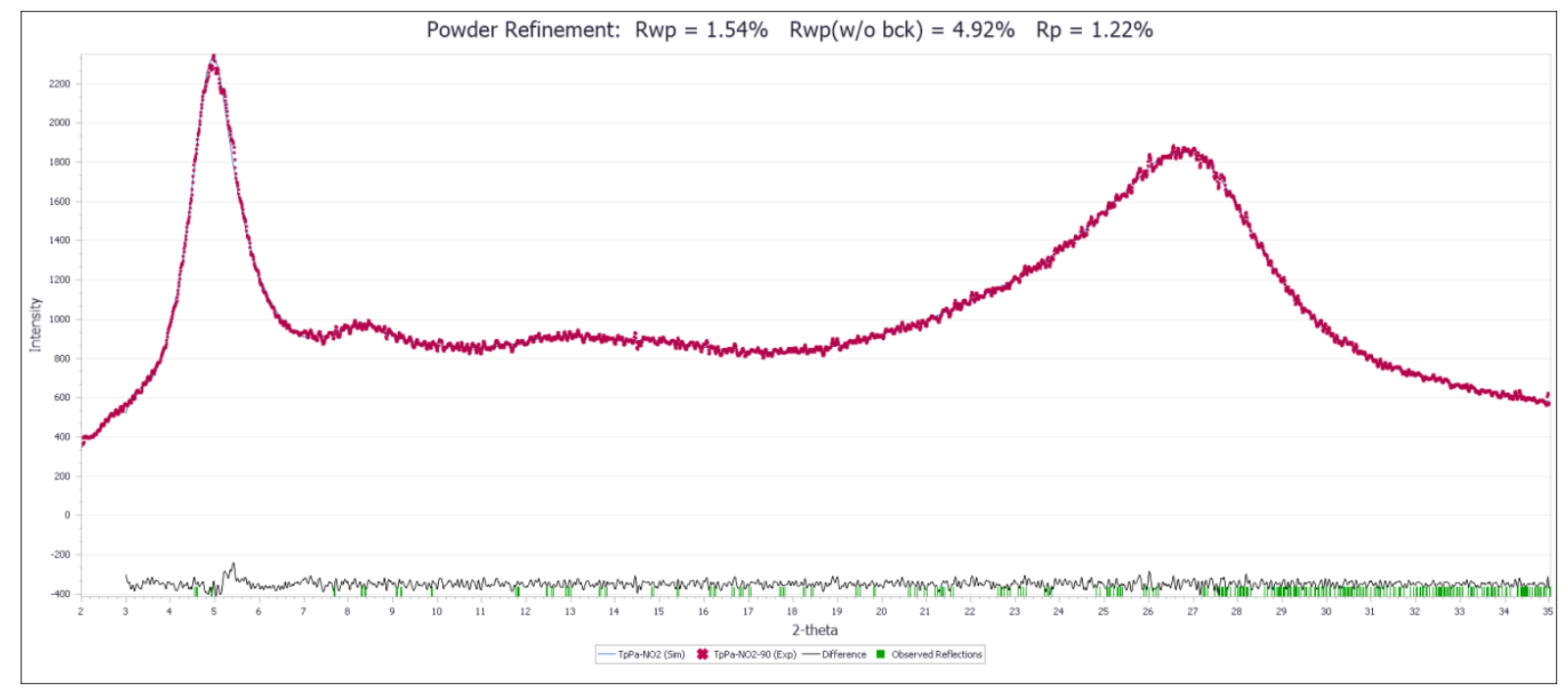

Figure S4 | Pawley refinement for TpPa-NO2-foam. Pawley refinement indicates the agreement between the simulated and experimental PXRD patterns. 


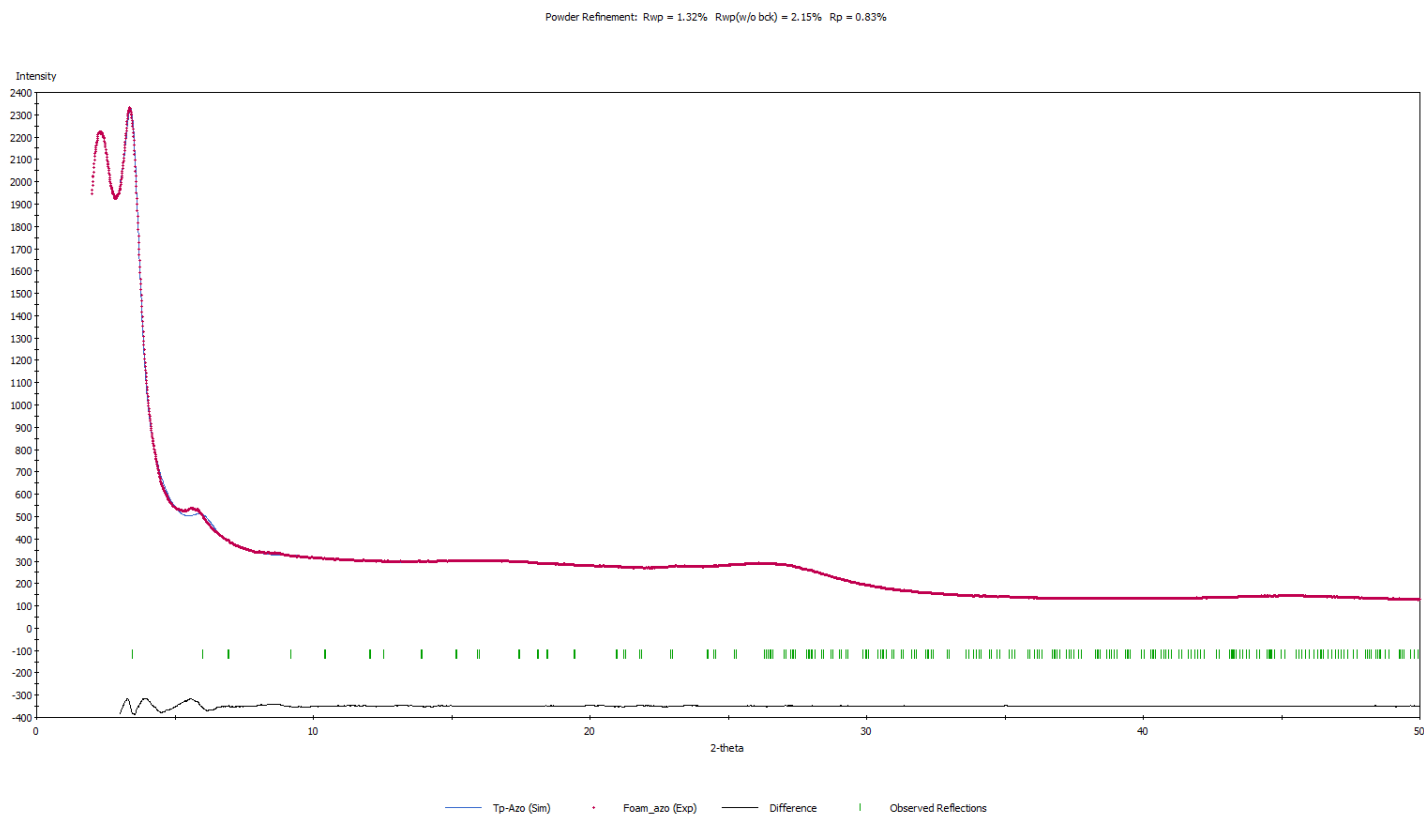

Figure S5 | Pawley refinement for TpAzo-foam. Pawley refinement indicates the agreement between the simulated and experimental PXRD patterns.

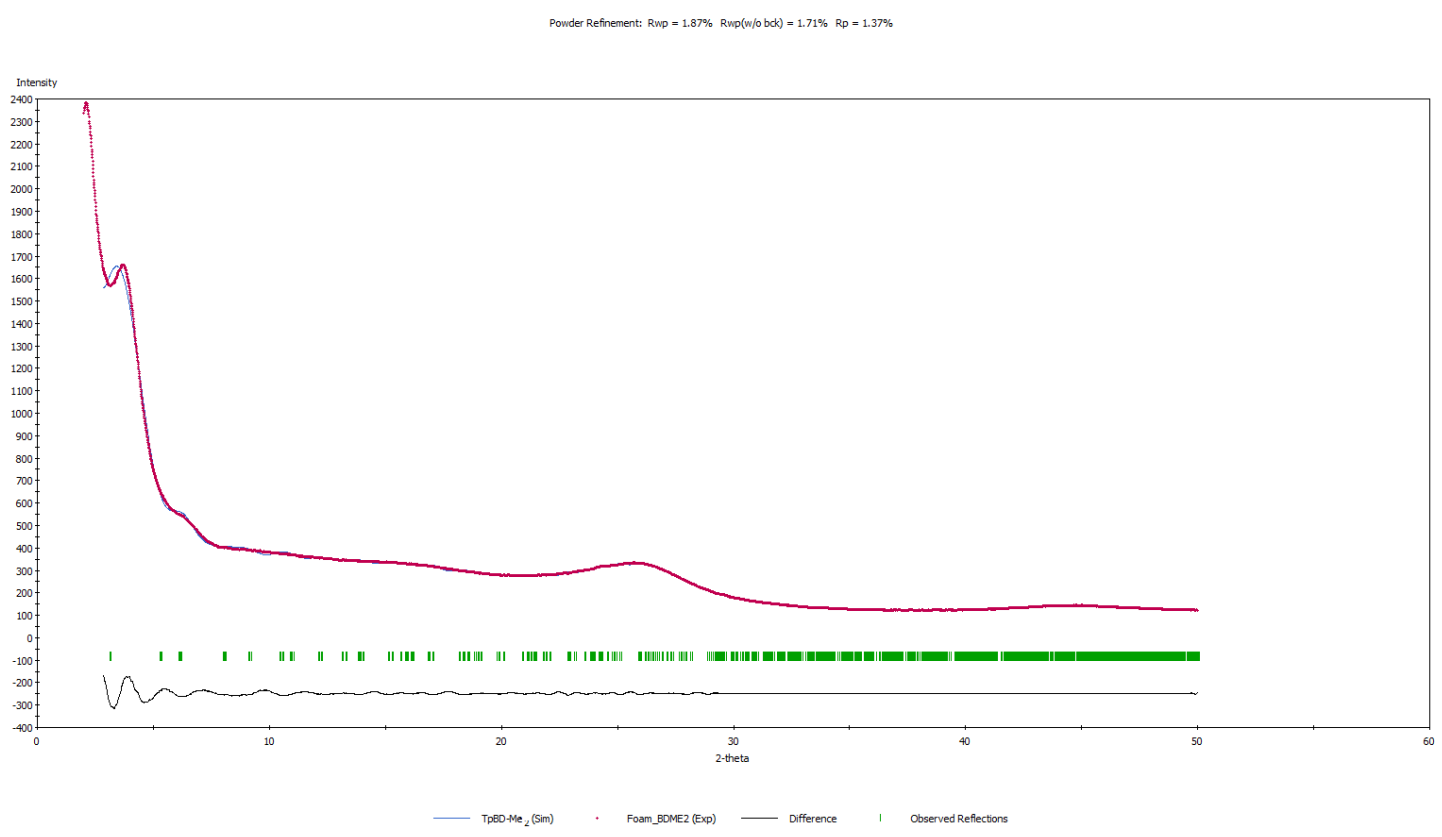

Figure S6 | Pawley refinement for TpBD-Me2-foam. Pawley refinement indicates the agreement between the simulated and experimental PXRD patterns. 


\section{Section S-4: FTIR spectra}
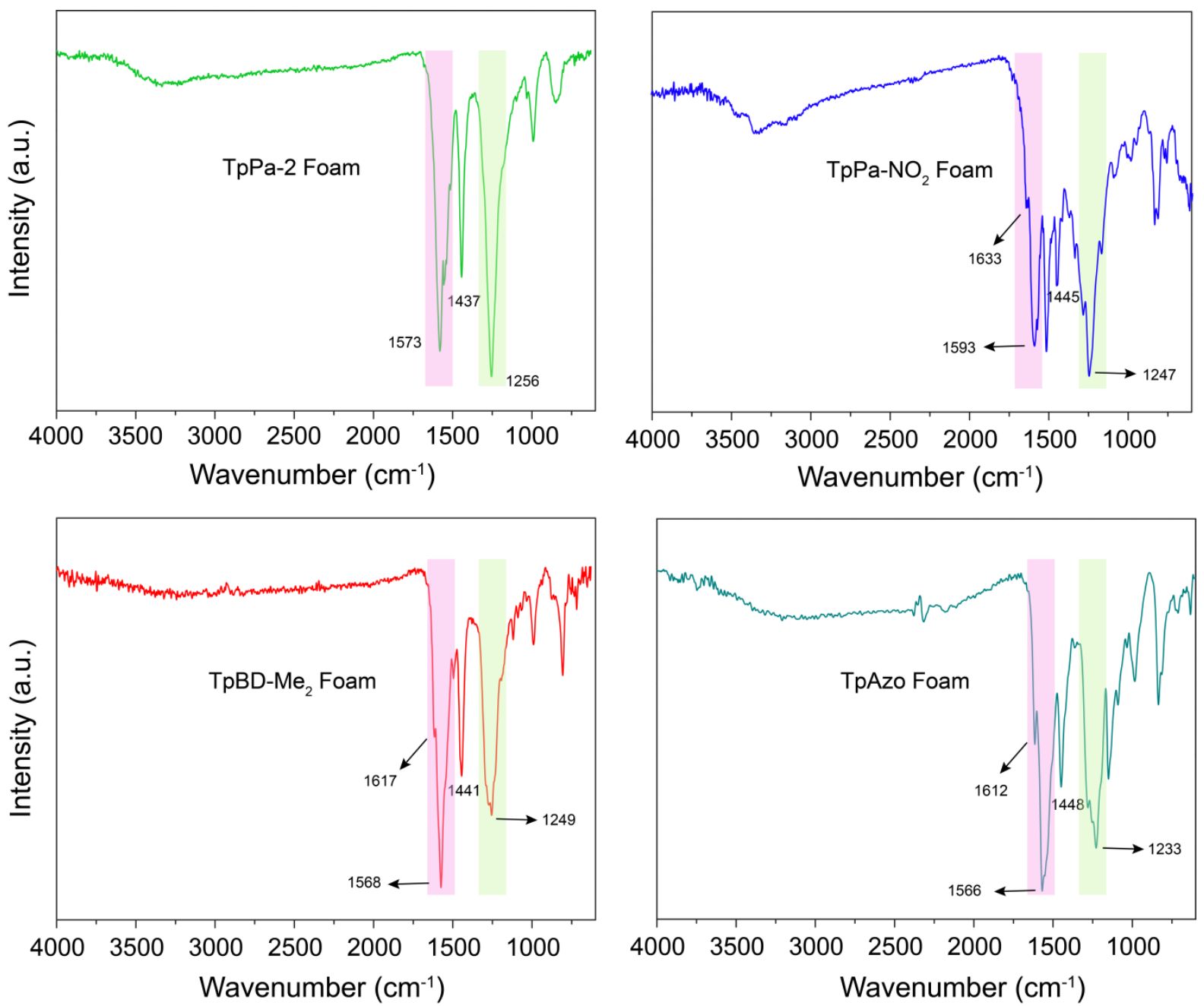

Figure S7 | FTIR spectra. FTIR spectra of the as-synthesized COF-foams show the characteristic peaks corresponding to $\beta$-ketoenamine-linked framework structures. Both $\mathrm{C}=\mathrm{C}$ and $\mathrm{C}-\mathrm{N}$ peak regions have been highlighted for ready reference. 


\section{Section S-5: ${ }^{13} \mathrm{C}$ CP-MAS spectra and TG analyses}
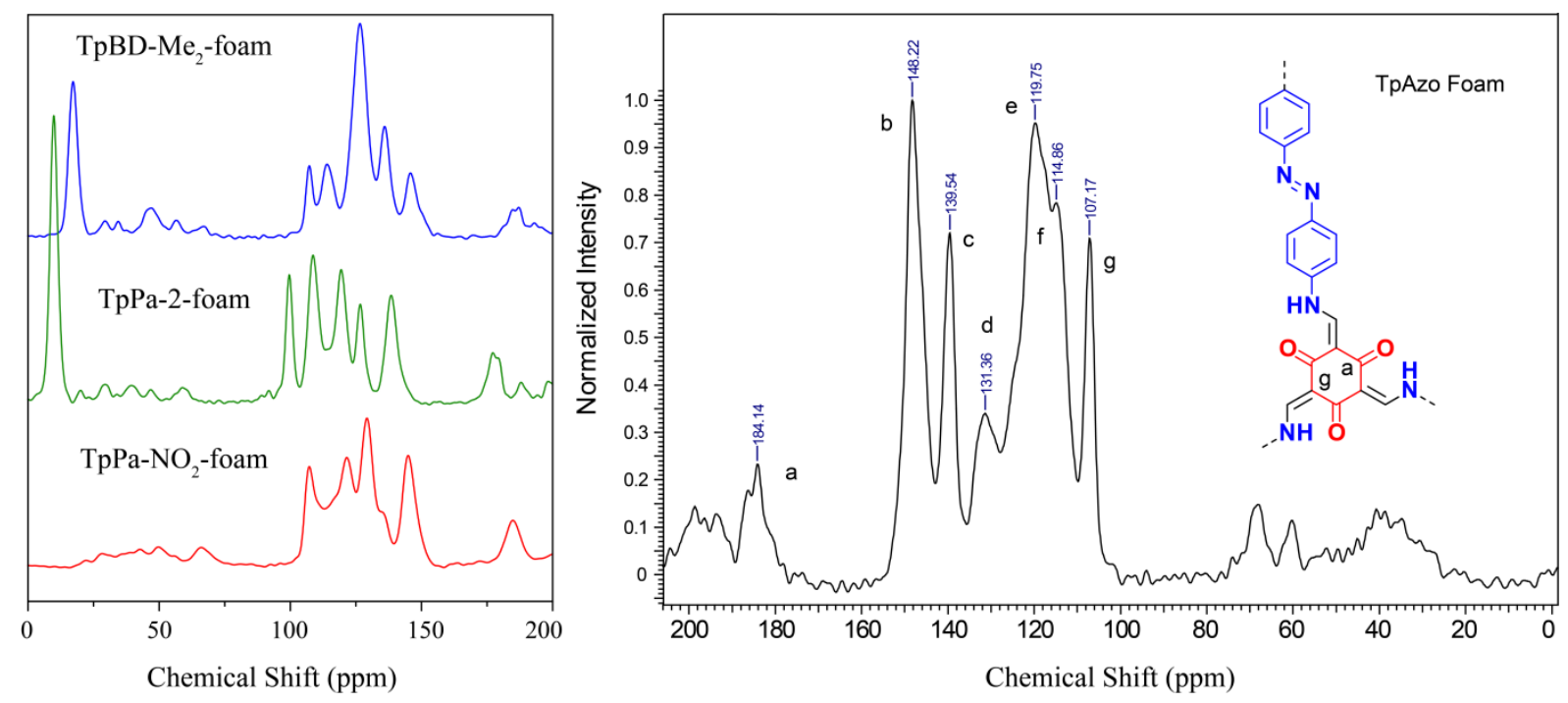

Figure S8 $\mid{ }^{13} \mathrm{C}$ CP-MAS spectra. Solid state ${ }^{13} \mathrm{C}$ CP-MAS spectra of the COF-foams show the characteristic carbon peaks. NMR also confirms the presence of $\beta$-ketoenamine-linkage within the framework.

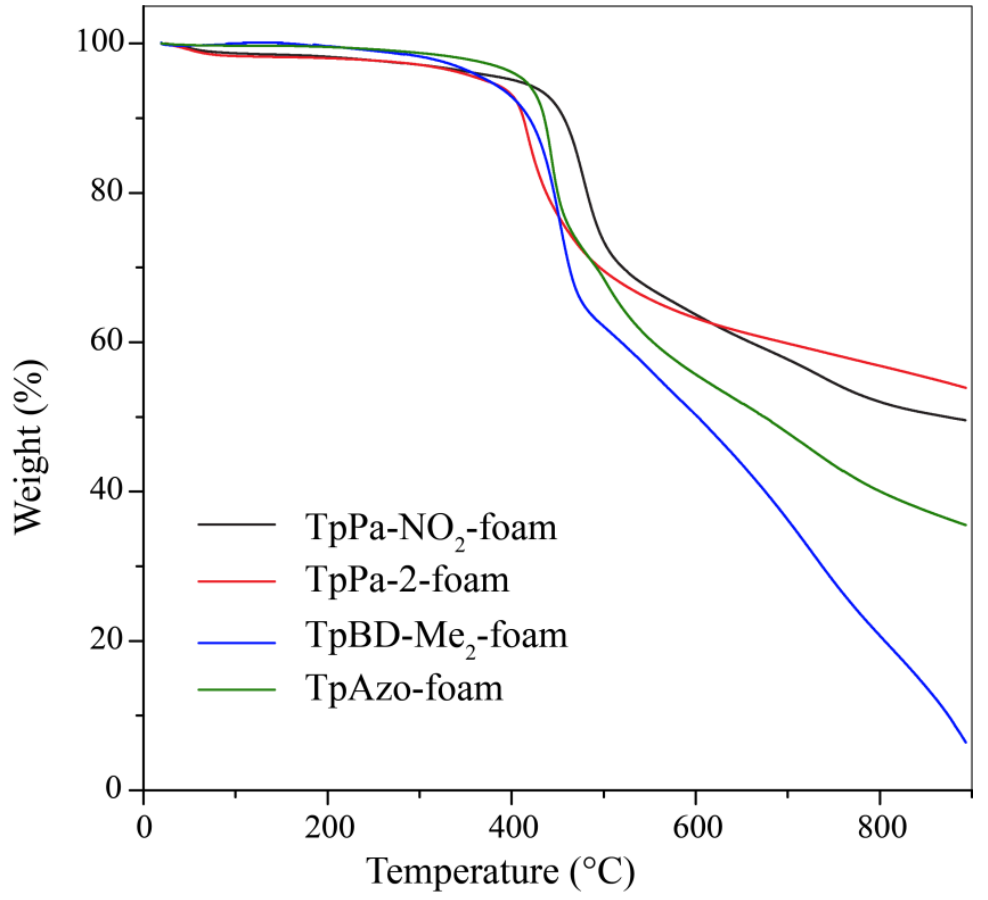

Figure S9 | TG analyses. TGA data of as-synthesized COF-foams confirm the high thermal stability (minimum up to $350{ }^{\circ} \mathrm{C}$ ) under $\mathrm{N}_{2}$ atmosphere. 


\section{Section S-6: SEM and TEM analyses}
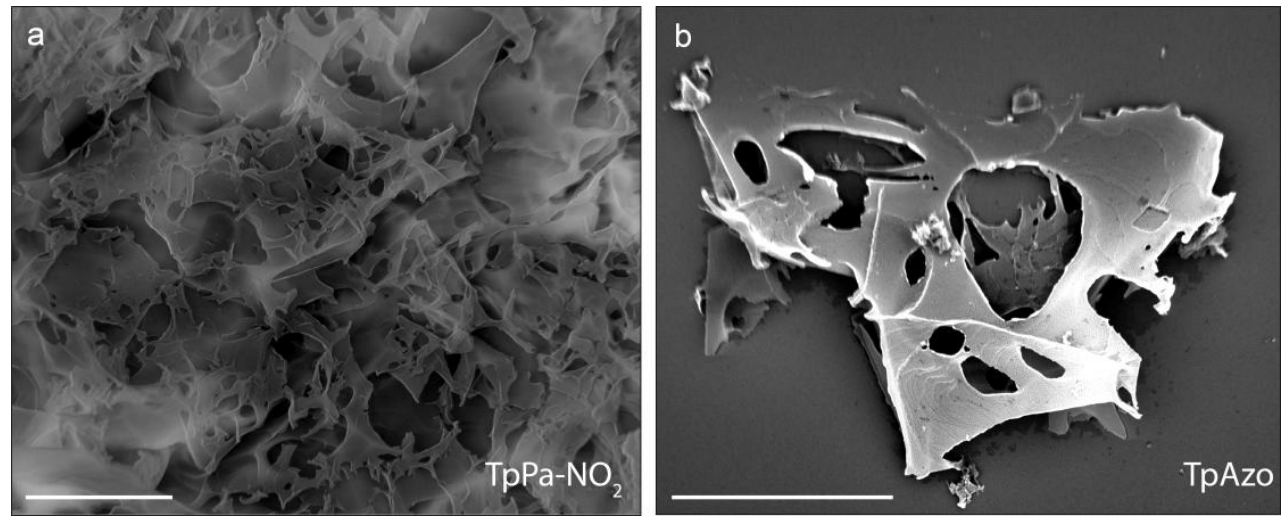

Figure S10 | SEM analyses of COF-foams. SEM images showing the random macro pores and defects within the a) TpPa-NO $25 \mu \mathrm{m})$, and b) TpAzo $(5 \mu \mathrm{m})$ (The numerical values in the brackets represent the scale bars for those respective foam).
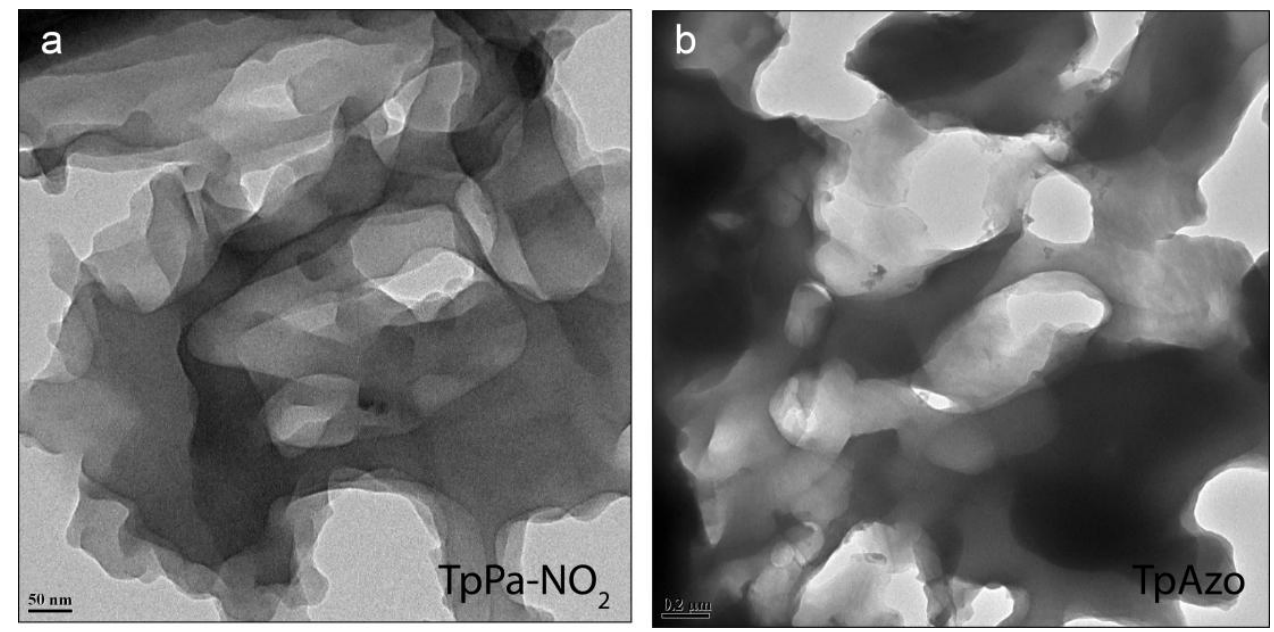

Figure S11 | TEM analyses of COF-foams. TEM images of the foam confirm the presence of large defects within the stacked framework structure (The numerical values in the brackets represent the scale bars for those respective images). 


\section{Section S-7: Micro-scale X-ray Computed Tomography and mechanical stability}

Three-dimensional imaging of COF-foam's was performed by X-ray microtomography using Xradia Versa 510 X-ray Microscope (Zeiss X-ray Microscopy, Pleasanton, CA, USA). COFfoam specimens were stained overnight with $5 \mathrm{wt} \%$ aqueous KI solution, loaded on to the sample holder. Sample holder is kept in between the X-ray source and detector assembly. Detector assembly consists of a scintillator, objective lens and a CCD camera. X-ray source is ramped up to $80 \mathrm{kV}$ and $7 \mathrm{~W}$. The tomographic image acquisitions were completed by acquiring 3201 projections over $360^{\circ}$ of rotation with a pixel size of 2.25 microns. In addition, projections without the samples in the beam (reference images) were also collected and averaged. Filtered back-projection algorithm is used for the reconstruction of the projections to generate two-dimensional (2D) virtual cross-sections of the specimens. Image processing software, Dragonfly Pro (Version 3.1) is used to generate volume-rendered threedimensional (3D) image of specimens.
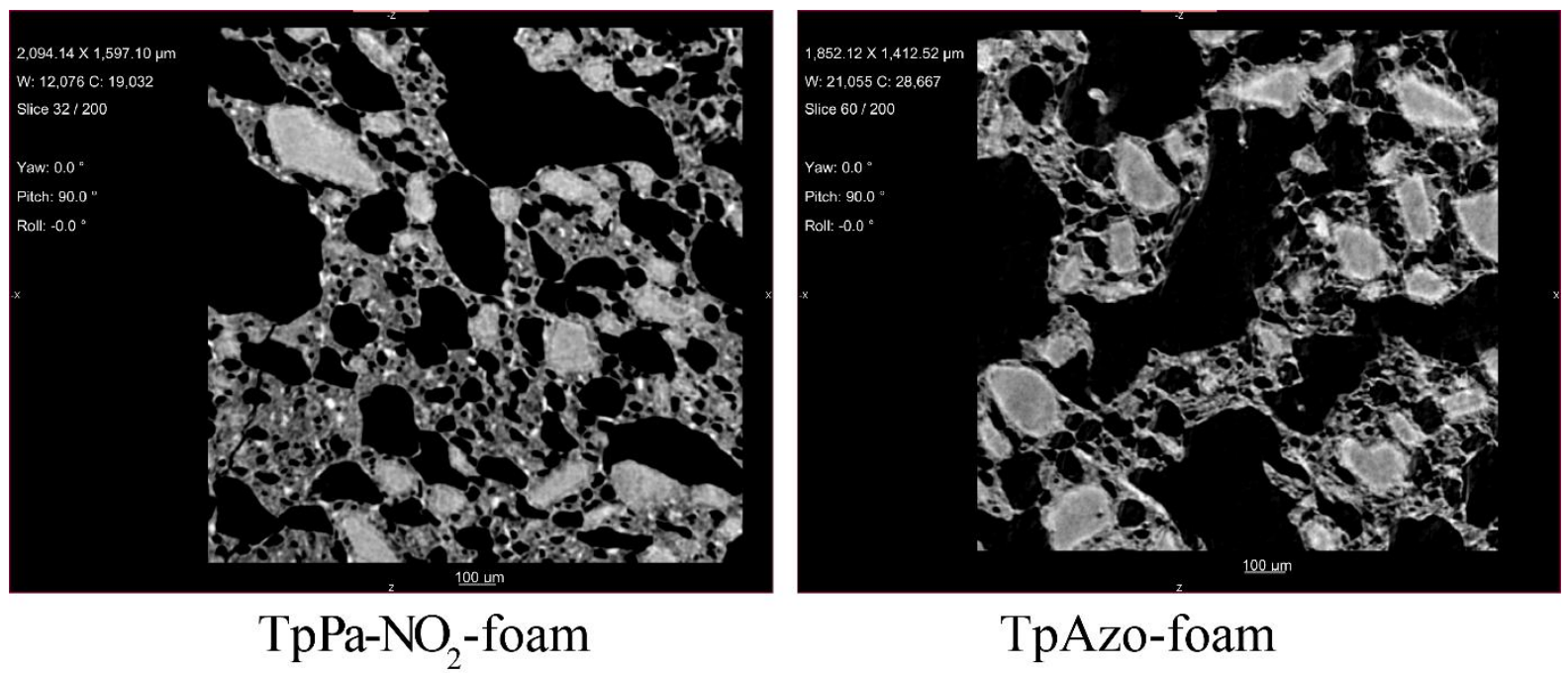

Figure S12 | Cross-sectional 2D tomography images. The cross sectional two dimensional tomography images of the foam confirm the presence of large of macropores of micrometer sizes within the nano-structure. 

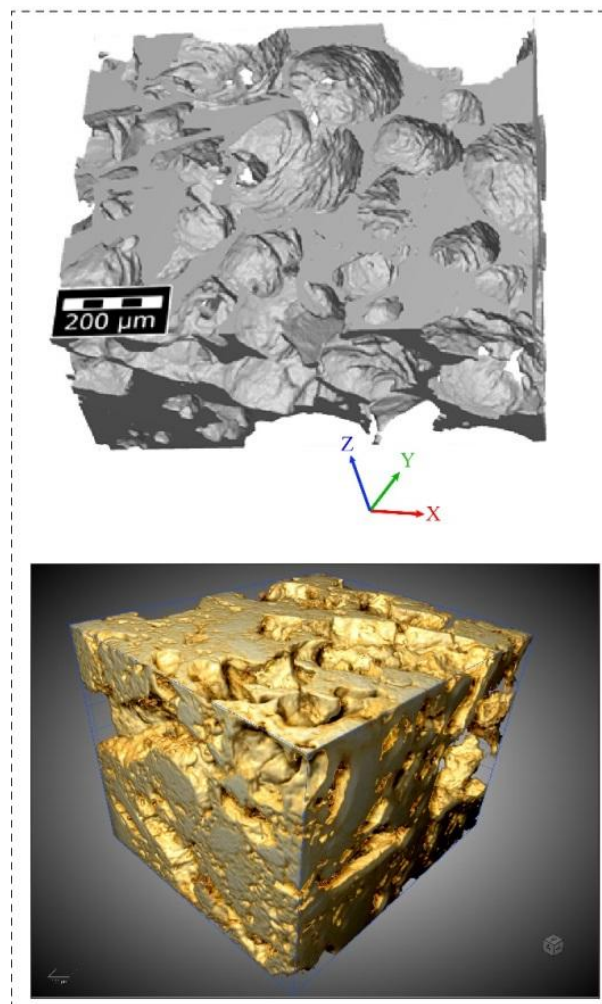

$\mathrm{TpPa}-\mathrm{NO}_{2}$-foam
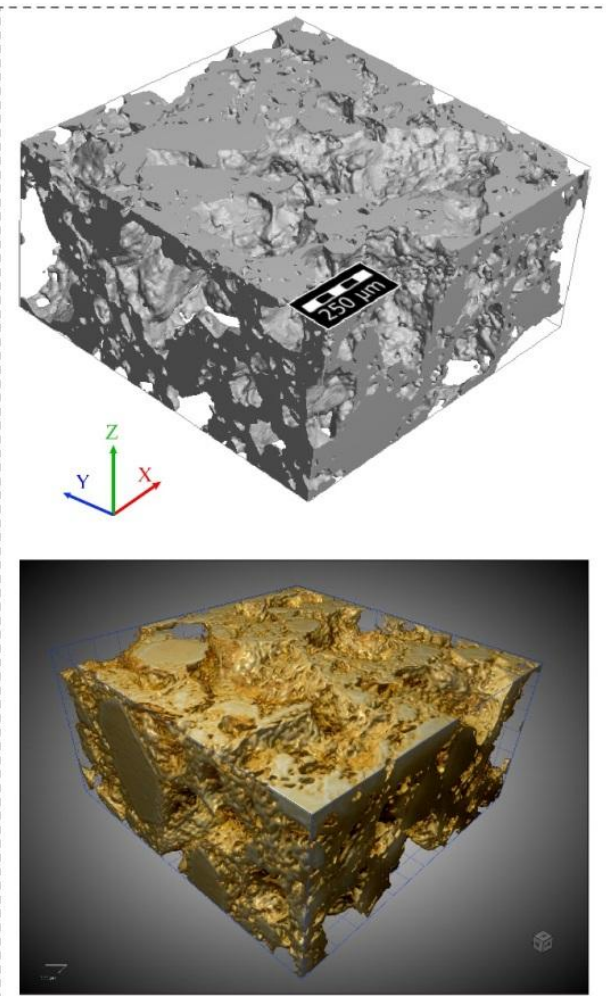

TpAzo-foam

Figure S13 | 3D volume-rendered images. The three dimensional volume rendered images clearly exhibits the defect within the ordered COF-foam. The defects are in macroscopic level.
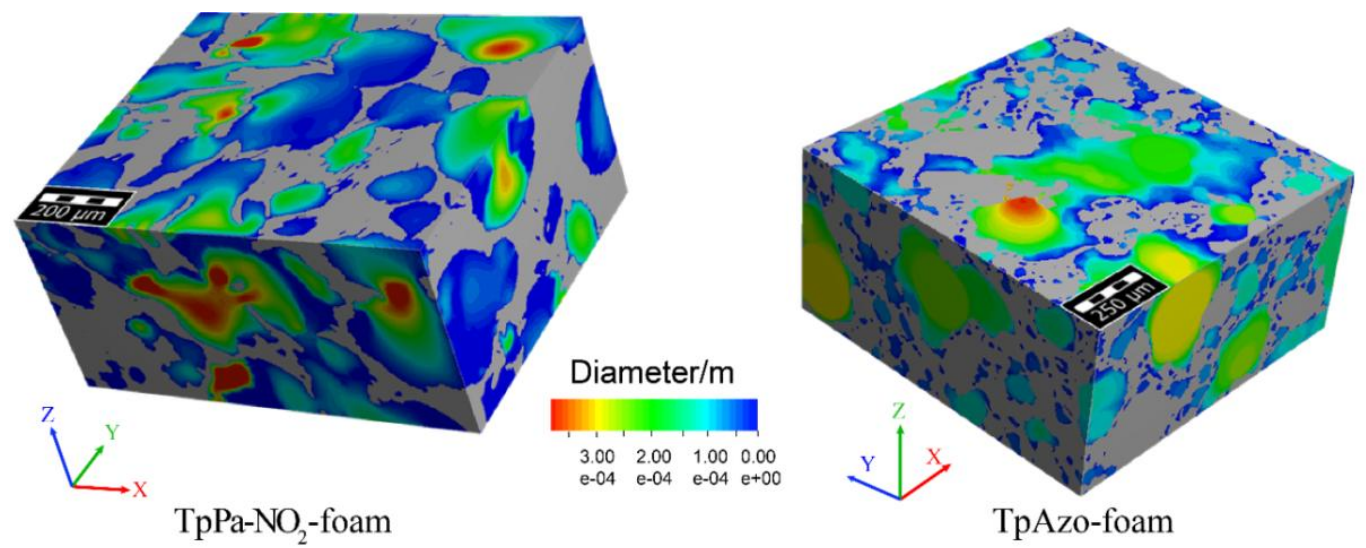

Figure S14 | Color-coded visualization of pore-size distribution. The three dimensional visualization of pore-size distribution reveal that various kind of pores are present inside the foam. Again, $99 \%$ of the total pores are interconnected. 

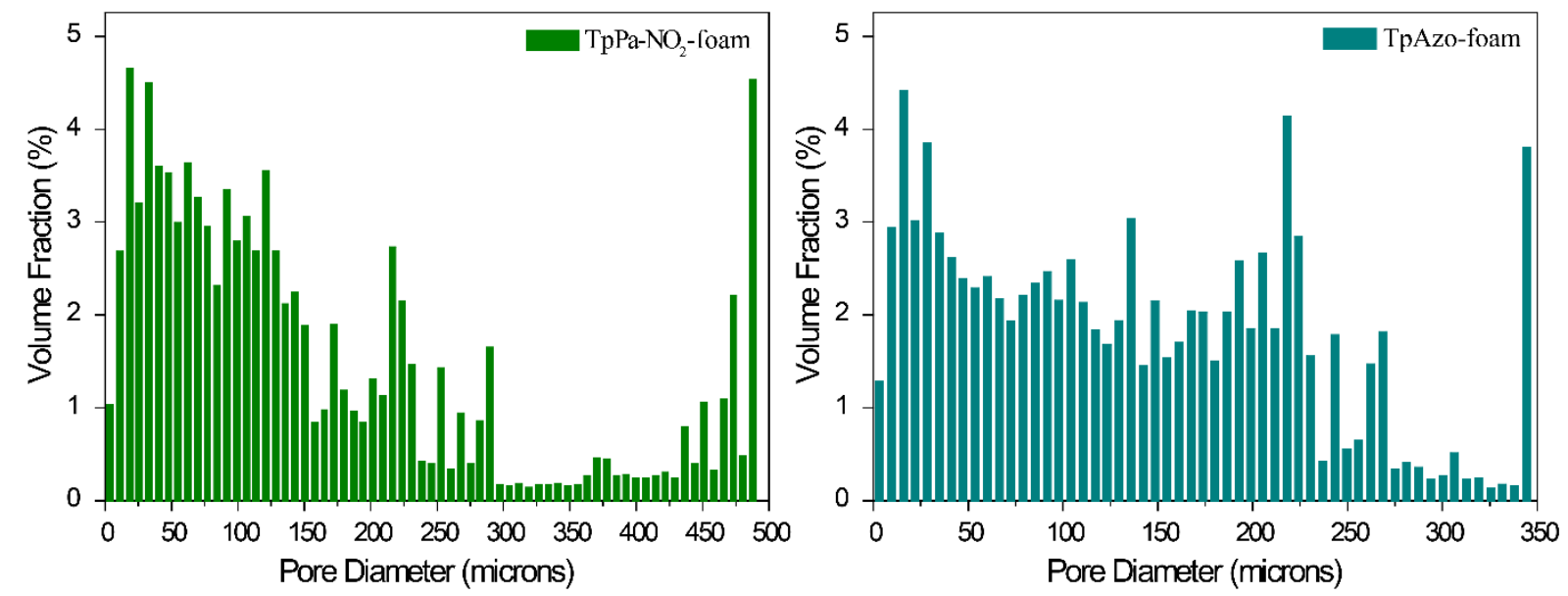

Figure S15 | Histogram of pore-size distribution. The histogram from X-ray computed tomography shows that there are various kind of pores within a foam block. It is due to the resolution limit, the macropores of $1 \mu \mathrm{m}$ and above have been represented herein. 

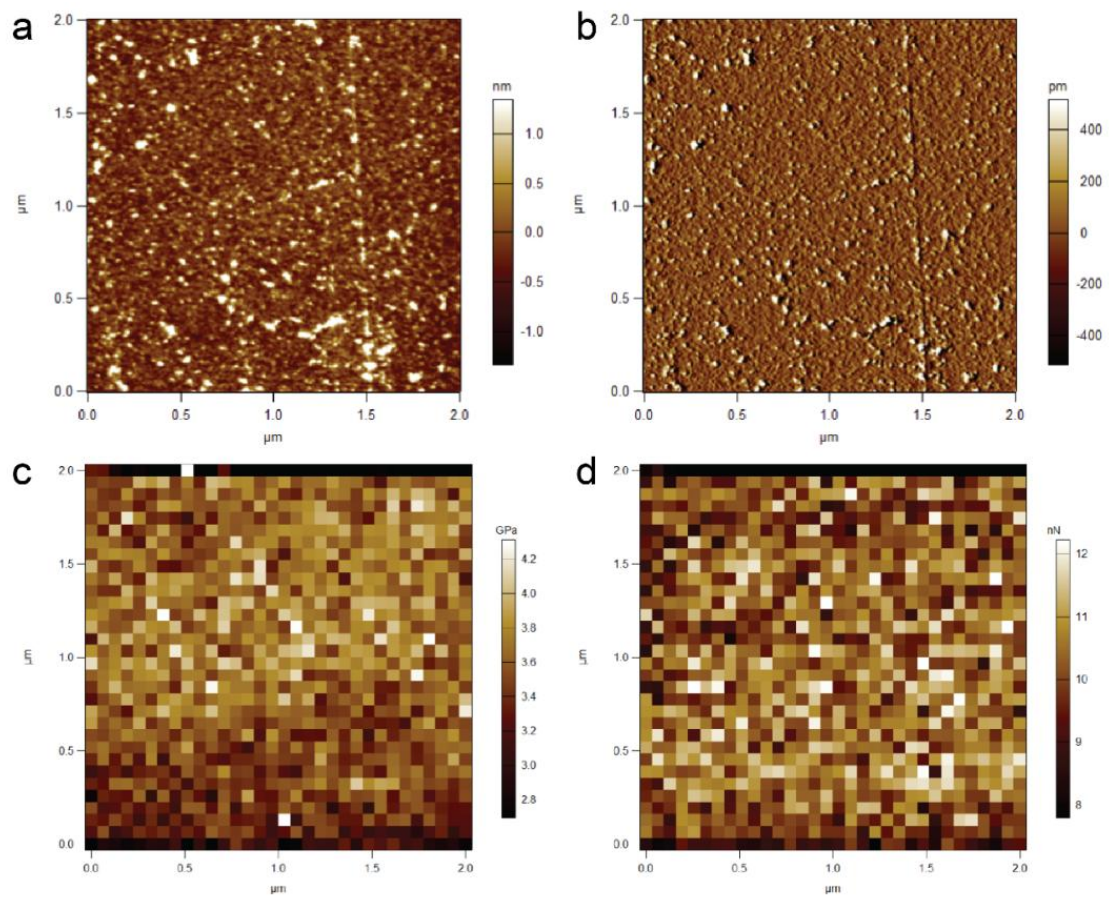

e

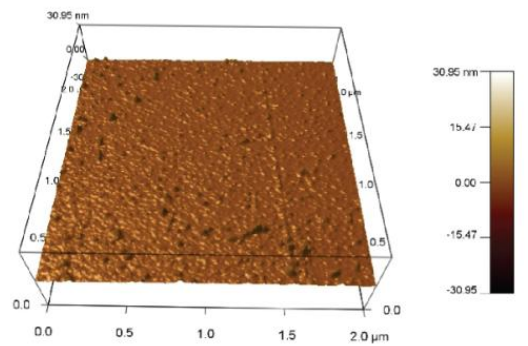

Invols: $60.44 \mathrm{~nm} / \mathrm{V}$ Spring Constant: $1.63 \mathrm{~N} / \mathrm{m}$ Trigger/force : $50 \mathrm{nN}$

Sample Modulus: 4.21 GPa Reduced $X_{V}^{2}$ : 980.658

Reduced Modulus: $4.70 \mathrm{GPa}$

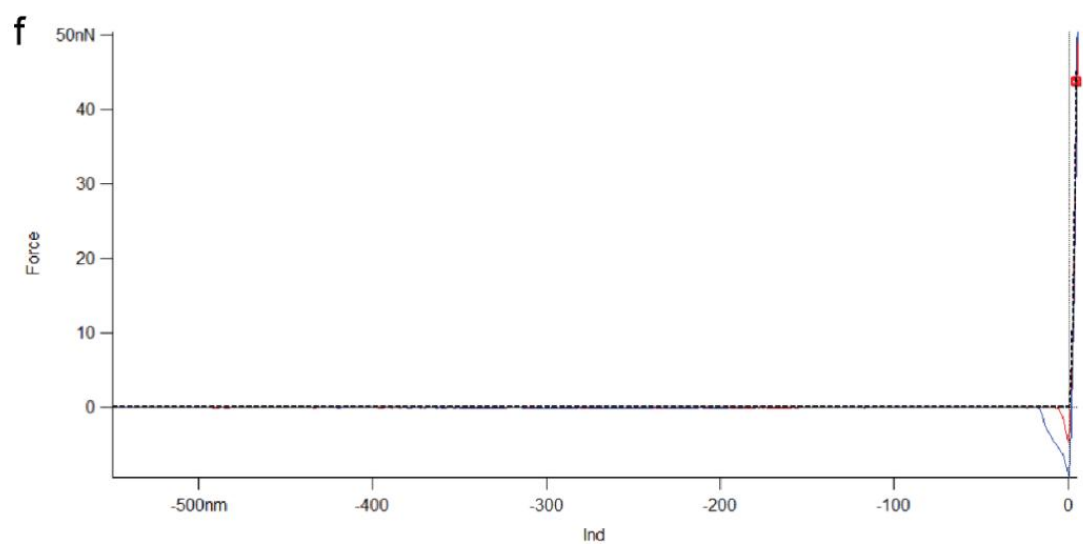

Figure S16 | Measurement of mechanical strength of the TpPa-2-foam from AFM. a) Height image, b) amplitude image c) adhesion image, d) modulus image, e) 3D height profile image and f) force curve of the as-synthesized TpPa-2-foam. The mechanical strength measurement carried out at a scanning range of $2 \mu \mathrm{m} \times 2 \mu \mathrm{m}$, shows the average value of Young's modulus of $4.21 \mathrm{GPa}$ for the selected area. 


\section{Section S-8: Nitrogen adsorption analysis}

BET theory is an extension of Langmuir theory, which gives the adsorption of gas molecule on monolayer's only. But BET theory explains the physical adsorption of gas molecules on a solid surface and serves as the basis for the measurement of the specific surface area of a material. The BET equation is,

$$
\frac{1}{v\left[\left(p_{0} p\right)-1\right]}=\frac{c-1}{v_{\mathrm{m}} c}\left(\frac{p}{p_{0}}\right)+\frac{1}{v_{\mathrm{m}} c}
$$

Where $p$ and $p_{0}$ are the equilibrium and the saturation pressure of adsorbents at the temperature of adsorption, $v$ is the adsorbed gas quantity (for example, in volume units), and $v_{m}$ is the monolayer adsorbed gas quantity, $c$ is the BET constant.

BJH method (Barrett, Joyner, and Halenda) has been used for calculating pore size distributions from experimental nitrogen adsorption isotherms using the Kelvin model of pore filling.
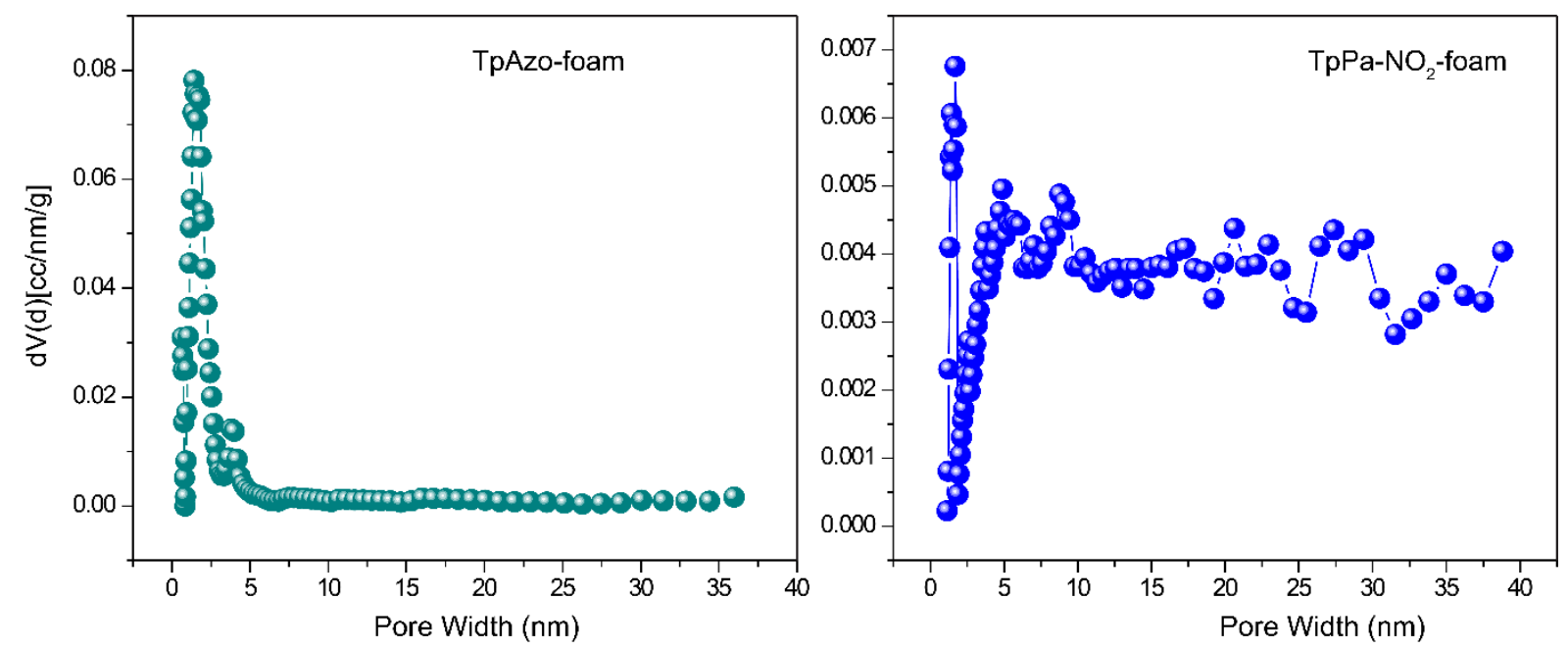

Figure S17 | Pore size distribution. Pore size distributions of COF-foams are calculated using the non-local density functional theory (NLDFT) model show different pore size distribution for COFs synthesized from various acid-diamine salts. 

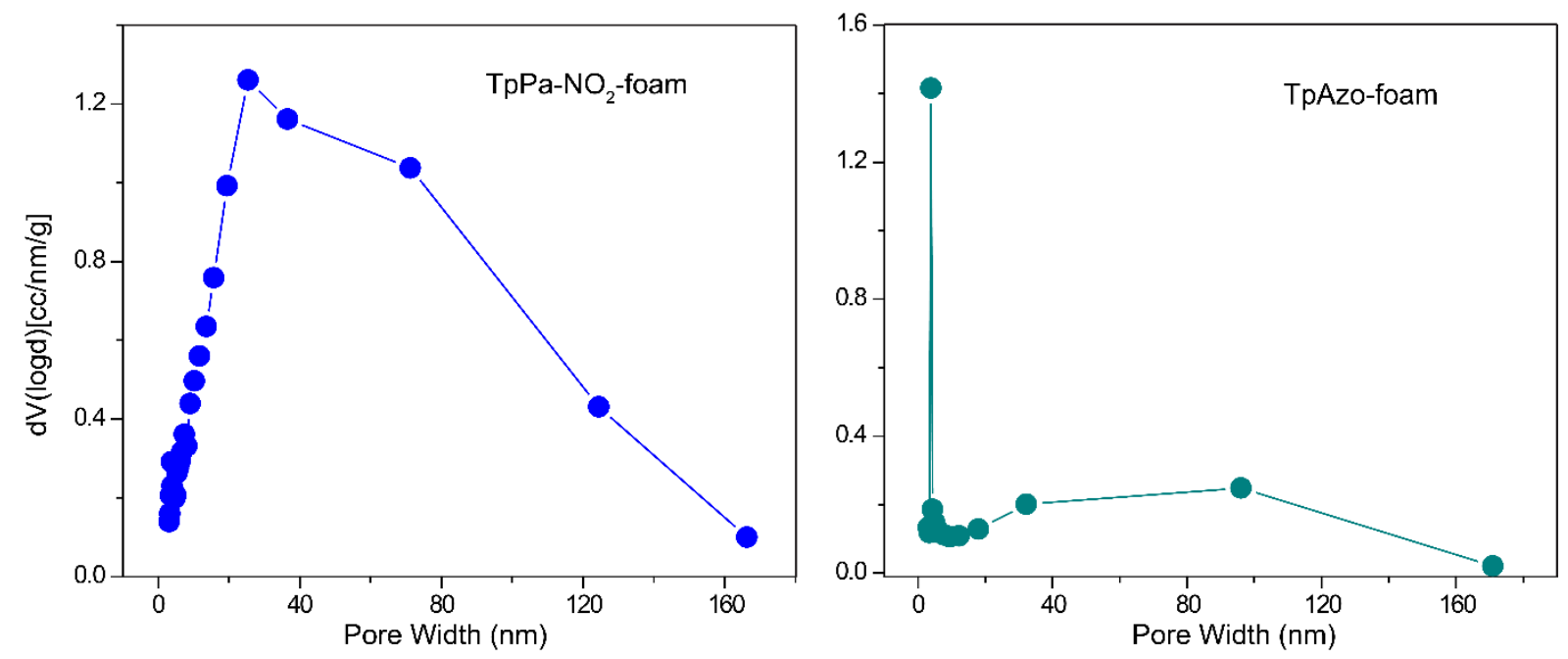

Figure S18 | Pore size distribution. Pore size distributions of COF-foams, represented herein, are calculated using the $\mathrm{BJH}$ model. A large distribution of pores confirm the presence of macropores. 


\section{Section S-9: Mechanism of Foam Synthesis}
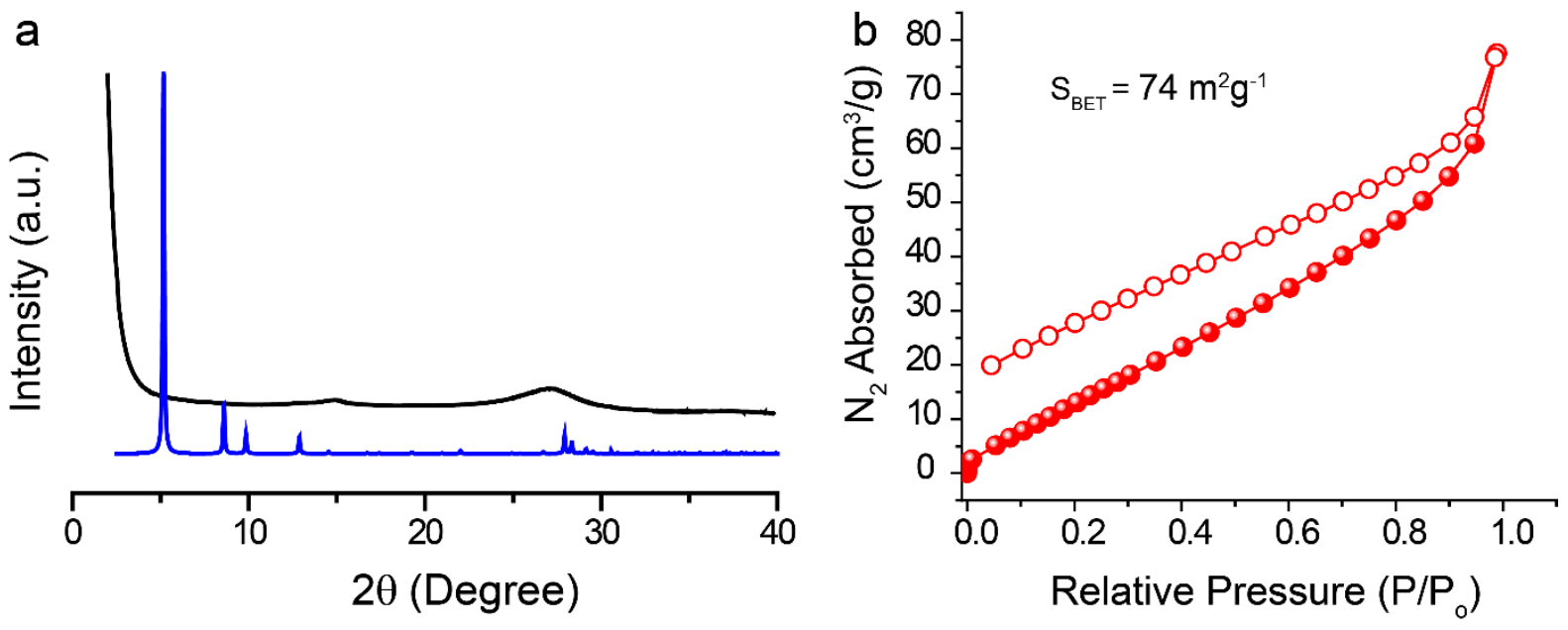

Figure S19 | Characterization of foams synthesized by non-conventional way. a) PXRD and b) gas adsorption show less crystallinity and poor surface area of the foam.

\section{Section S-10: Adsorption of pollutants}

Kinetic adsorption studies were performed with $10 \mu \mathrm{M}$ of pollutant stock solution. However, it is due to very low intense intense absorption maximum, Iodine stock was prepared at 100 $\mu \mathrm{M}$ of concentration. Again, Bisphenol A was also prepared of higher concentration of 25 $\mu \mathrm{M}$ considering the low intense absorption peak. For the maximum uptake capacity measurement of methylene blue, various concentration of stock solution was prepared. However, it is due to the very high intensity of absorption maxima, we were unable to calculate the absorbance of high concentration methylene blue solution. Thus, the intensity maxima of the stock solution were measured by multiplying with the low concentration absorption maxima with certain values. 

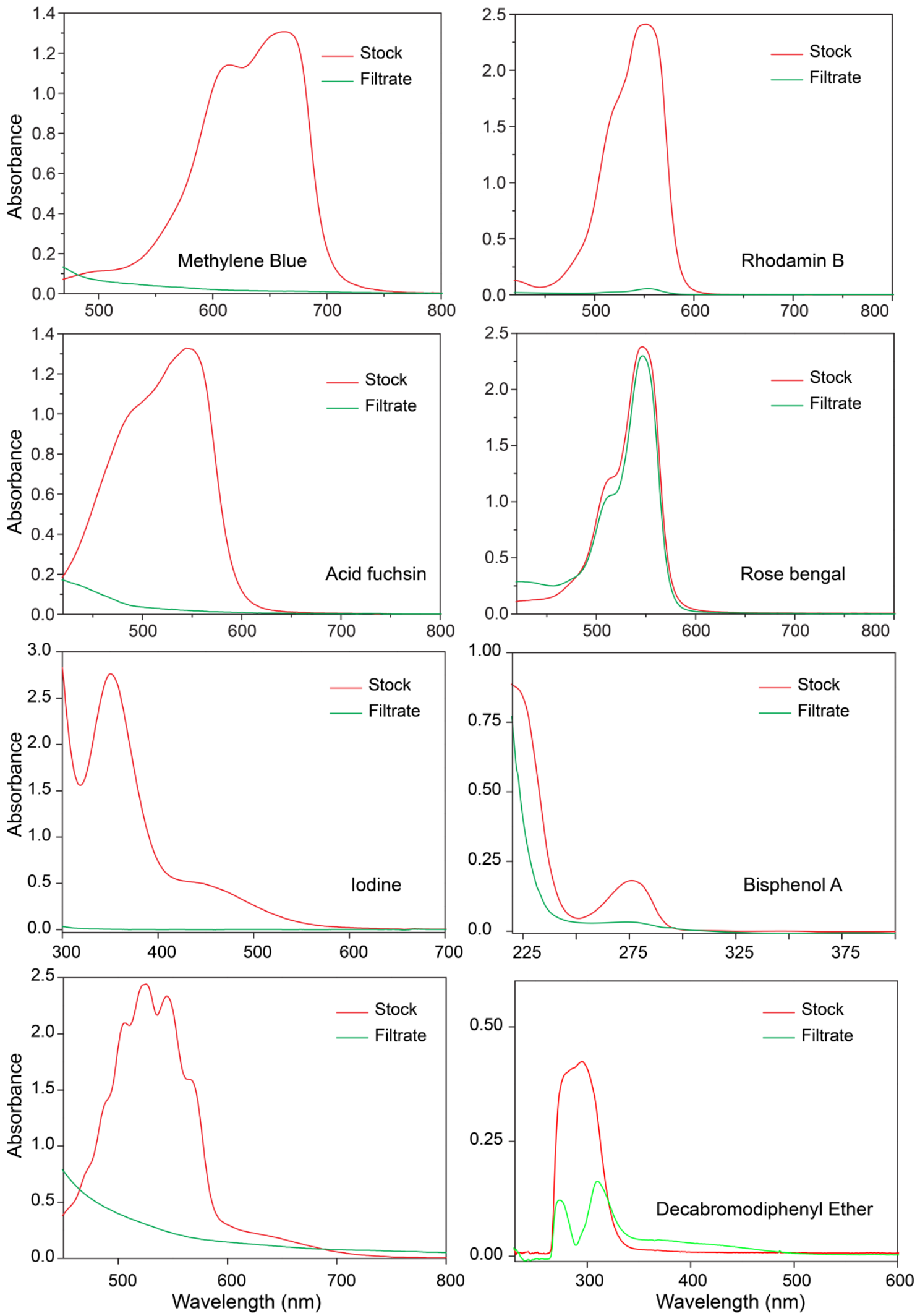

Figure S20 | Pollutant removal efficiency. Pollutant removal efficiency of TpPa-2-foam has been represented here in. 

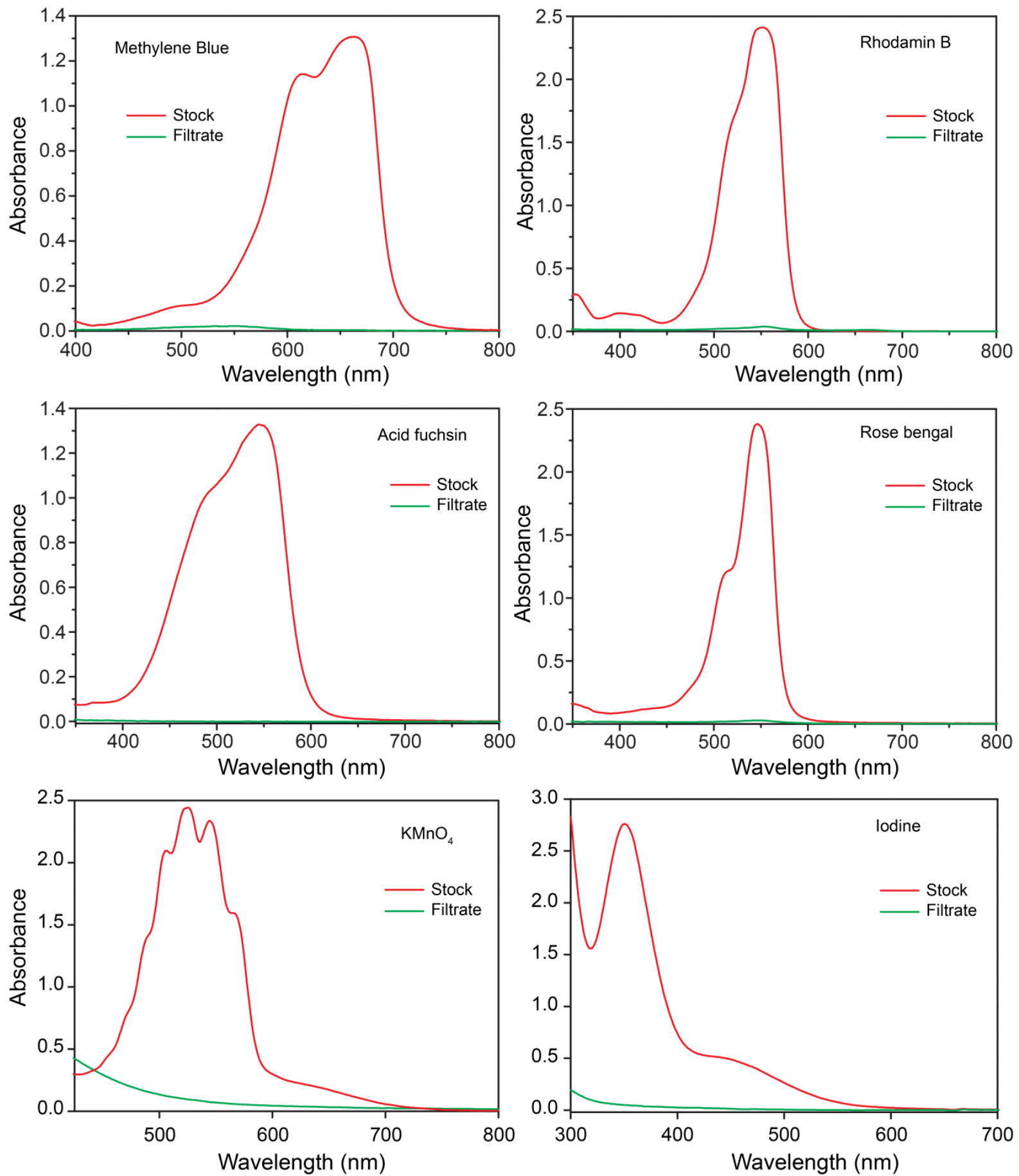

Figure S21 | Pollutant removal efficiency. Pollutant removal efficiency of $\mathrm{TpPa}-\mathrm{NO}_{2}$-foam has been represented here in. 

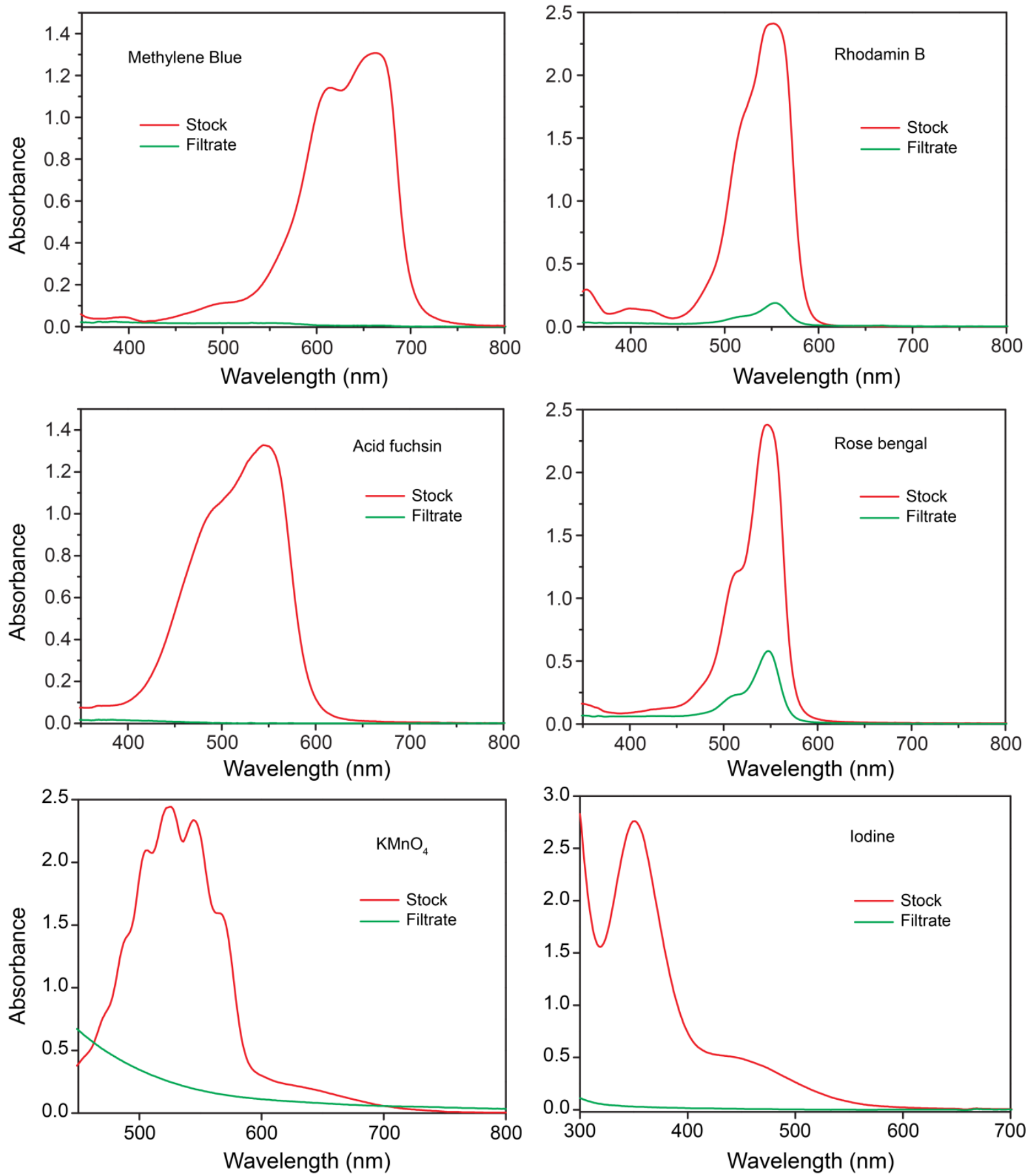

Figure S22 | Pollutant removal efficiency. Pollutant removal efficiency of TpAzo-foam has been represented here in. 

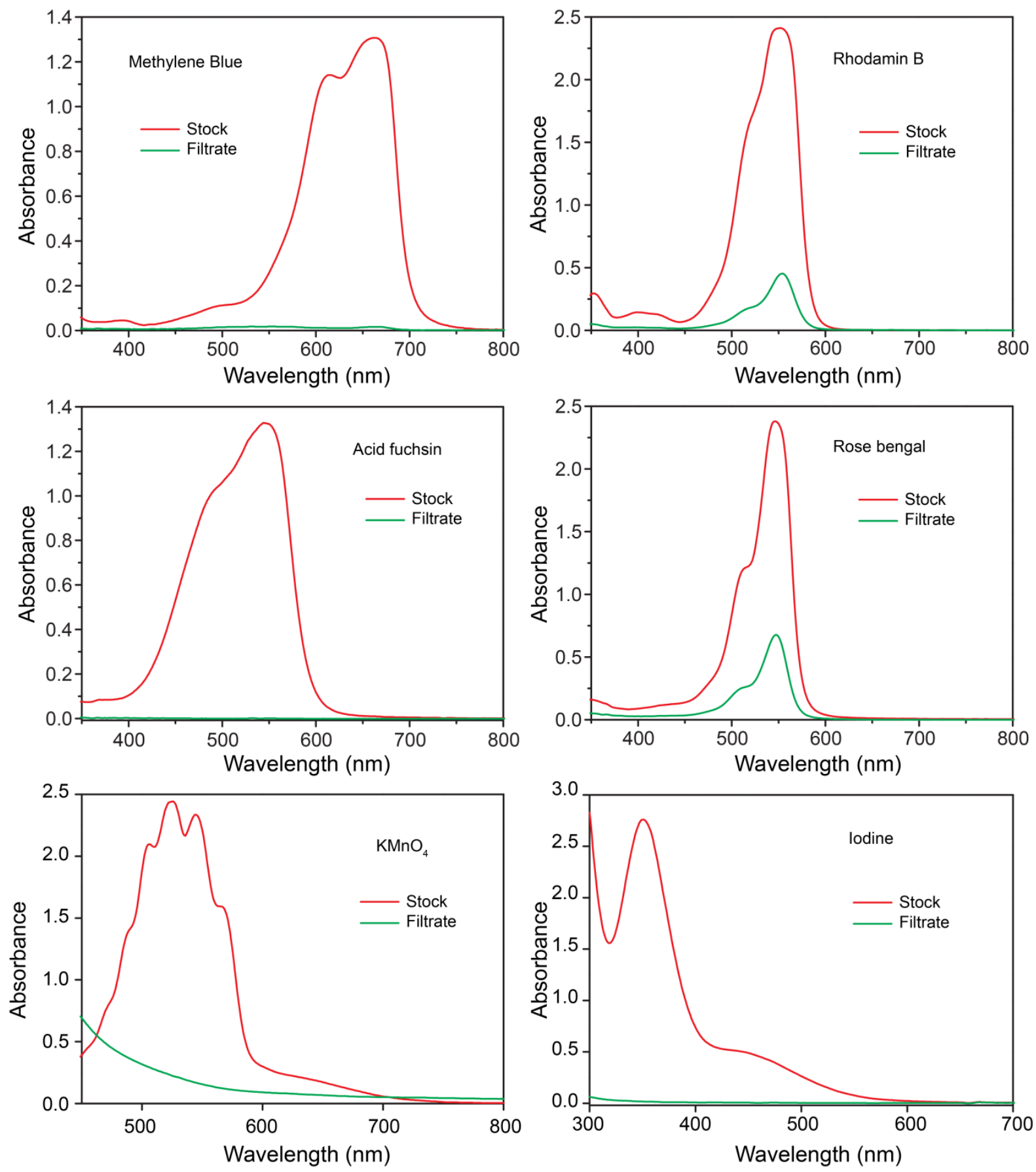

Figure S23 | Pollutant removal efficiency. Pollutant removal efficiency of TpBD-Me 2 -foam has been represented here in. 


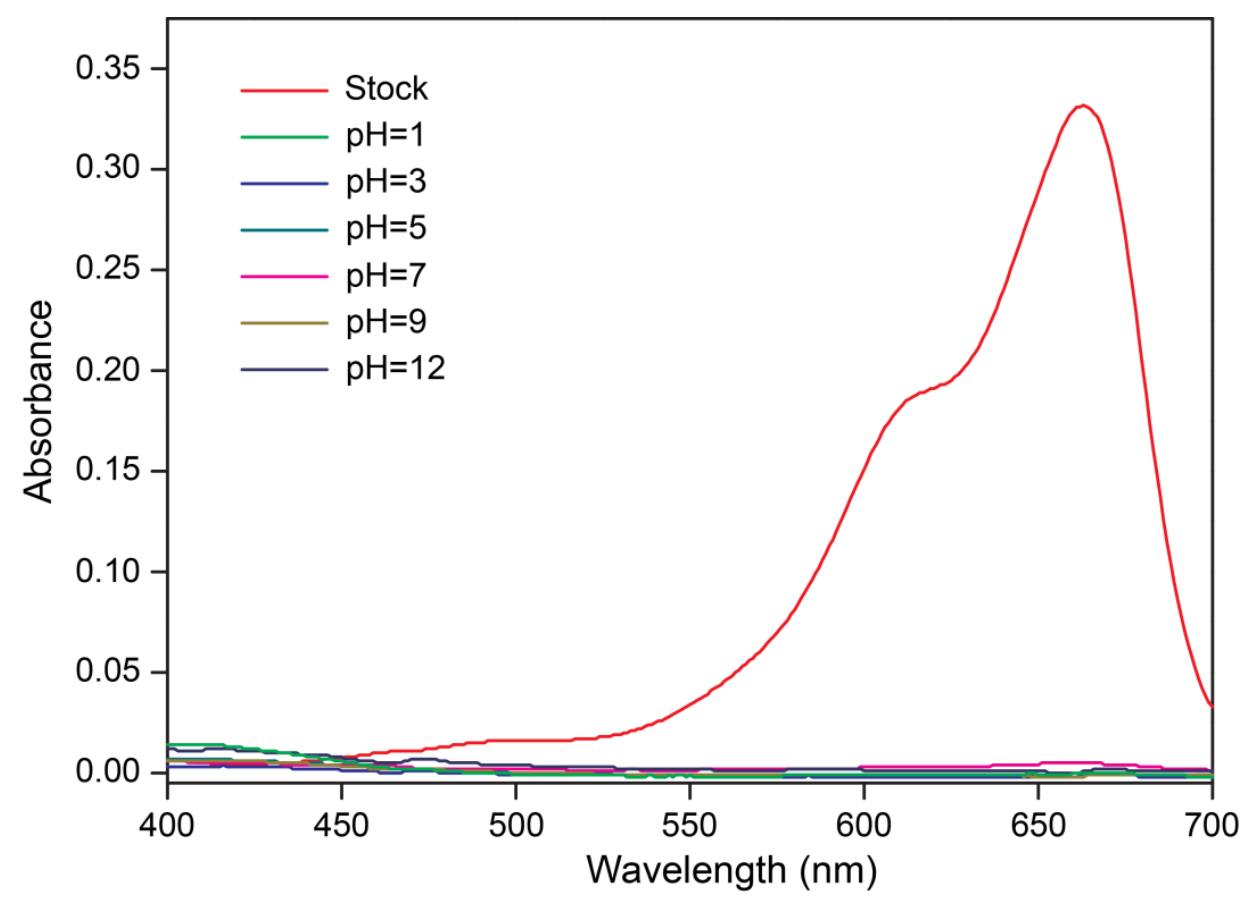

Figure S24 | pH dependent removal efficiency. The pollutant removal efficiency at various $\mathrm{pH}$ has been verified with high removal efficiency of MB pollutant.

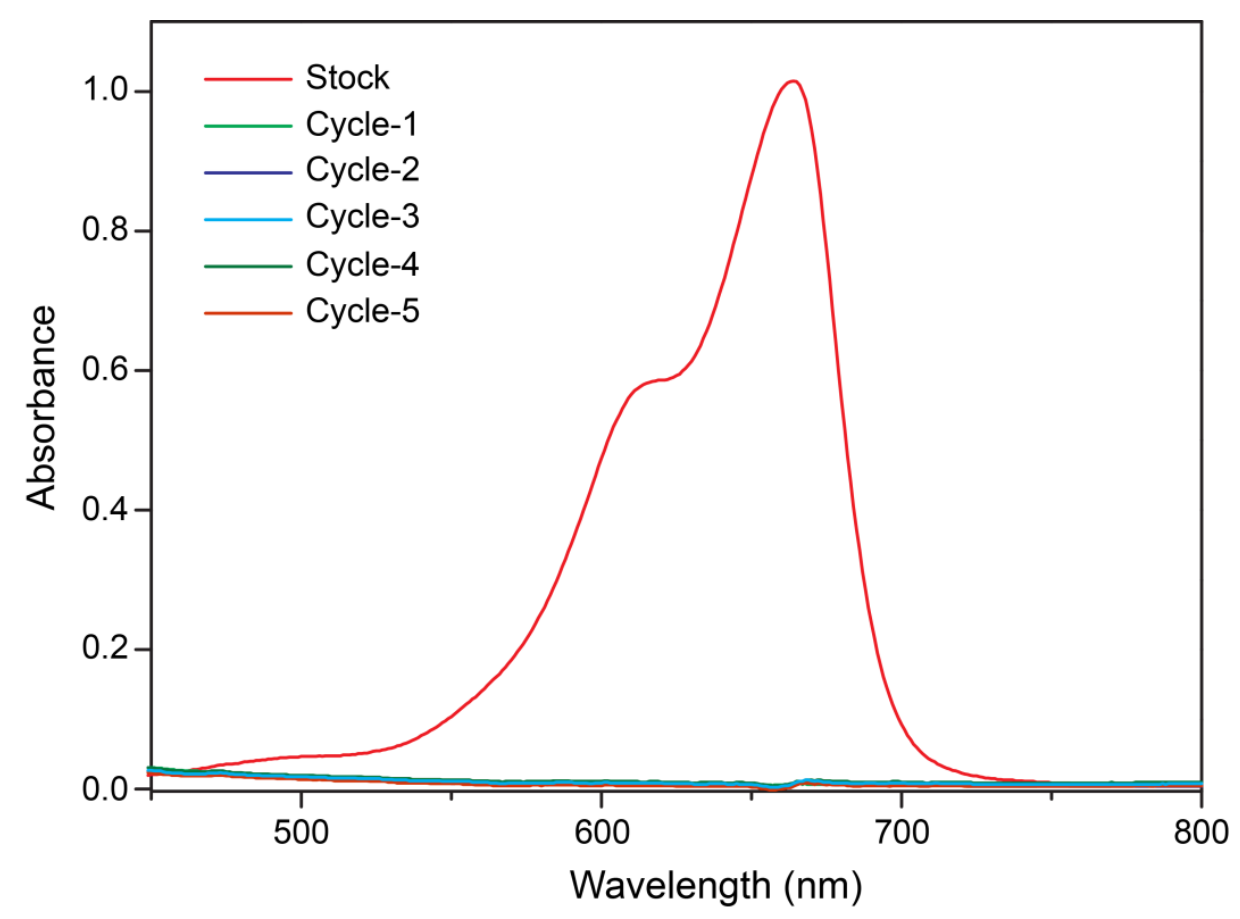

Figure S25 | Recyclability test. The recyclability of TpPa-2 foam has been performed with MB dye. The measurement clearly reveals that there is no compromise with the performance. 

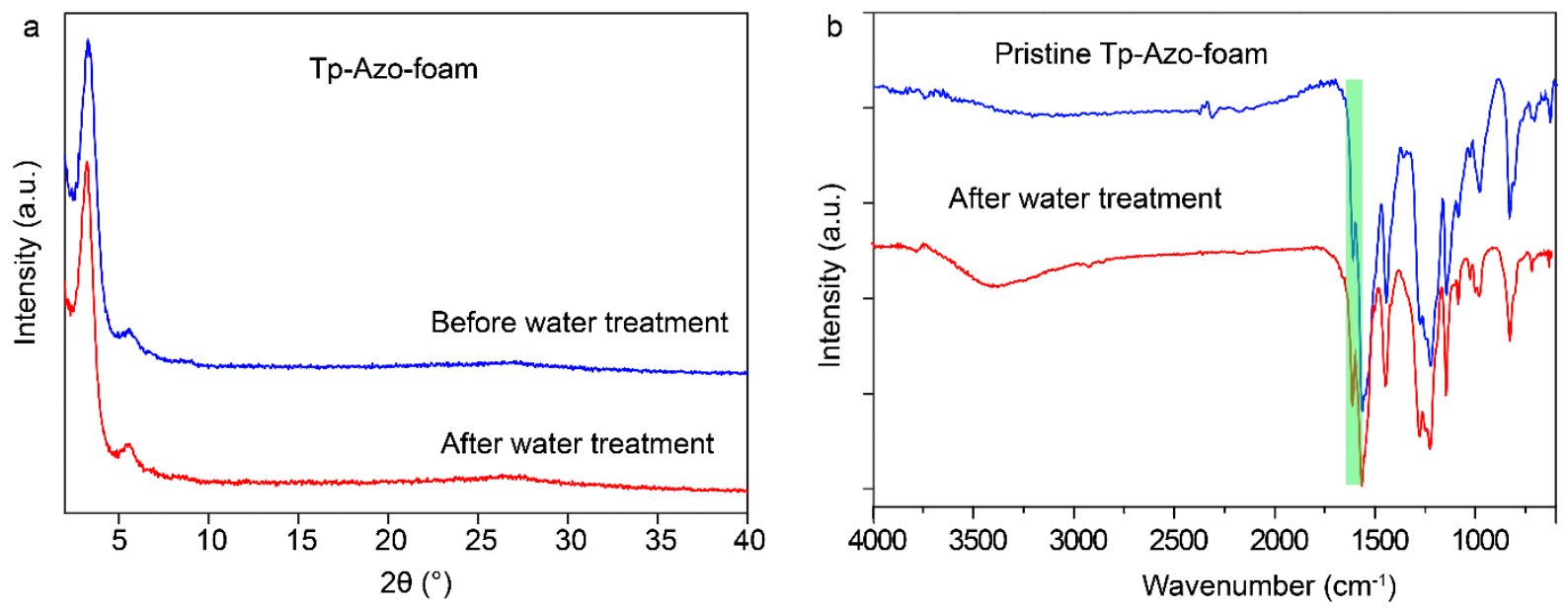

Figure S26 | Water stability of foam. Comparison of a) PXRD and b) IR patterns of foam before and after the water treatment.

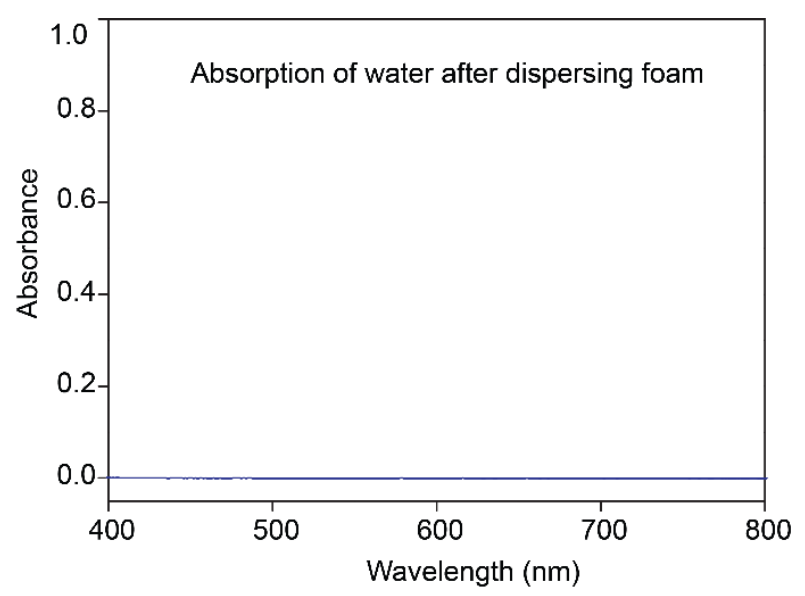

Figure S27 | Water stability of foam. UV-vis absorption spectrum of the water after treating foam for one day. No absorption in the UV-vis range suggests that there is no secondary pollution from the adsorbent. 

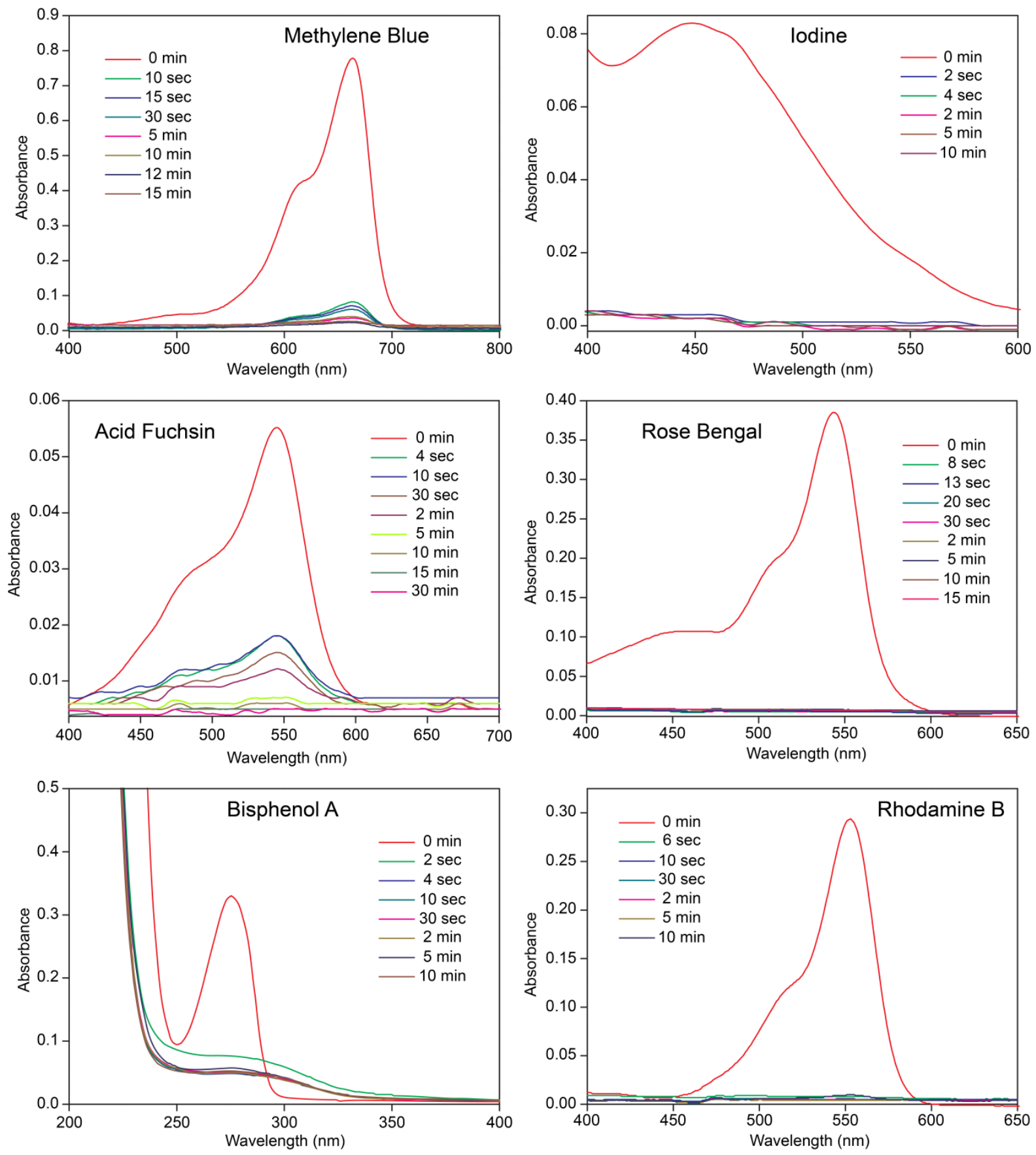

Figure S28 | Kinetic adsorption measurement. Time-dependent removal efficiency of pollutants has been measured for TpPa-2-foam. 

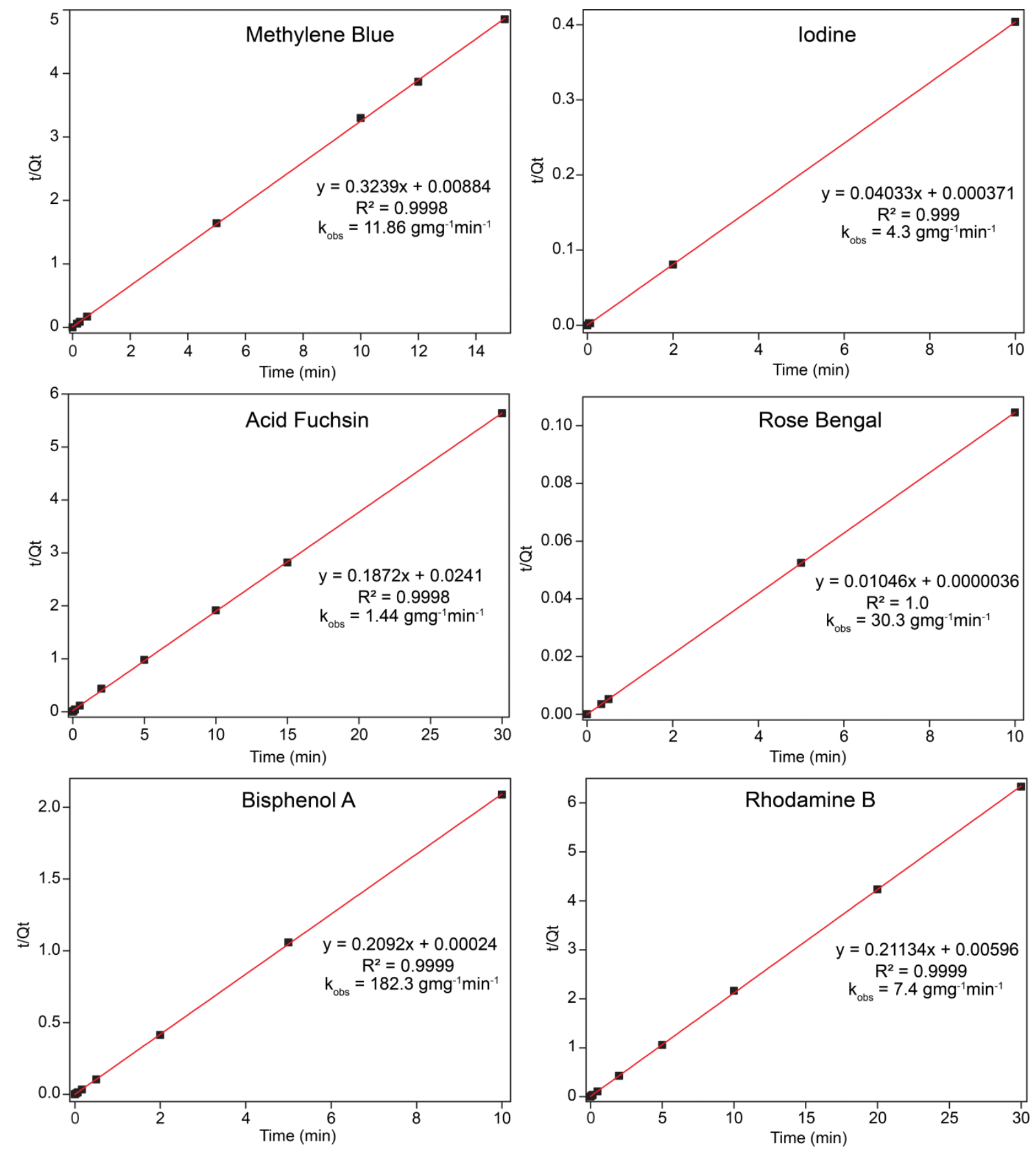

Figure S29 | Kinetic adsorption measurement. The rate of adsorption has been measured from the linear fit Langmuir plot for TpPa-2-foam for the adsorption of pollutants. 
Table S3.| Rate of adsorption of Bisphenol A by various adsorbents.

\begin{tabular}{|c|c|c|}
\hline Sorbent & $\boldsymbol{k}_{\text {obs }}\left(\mathbf{g ~ m g}^{\mathbf{- 1}} \mathbf{~ m i n}^{-\mathbf{1}}\right)$ & Correlation Coefficient $\mathbf{R}^{\mathbf{2}}$ \\
\hline P-CDP & 1.5 & 1.00 \\
\hline NP-CDP & 0.10 & 1.00 \\
\hline EPI-CDP & 0.032 & 1.00 \\
\hline NAC & 0.090 & 1.00 \\
\hline GAC & 0.047 & 0.96 \\
\hline Brita AC & 0.0088 & 0.99 \\
\hline CalPn & 2.12 & 0.99 \\
\hline TpPa-2-foam & 182.3 & \\
\hline
\end{tabular}
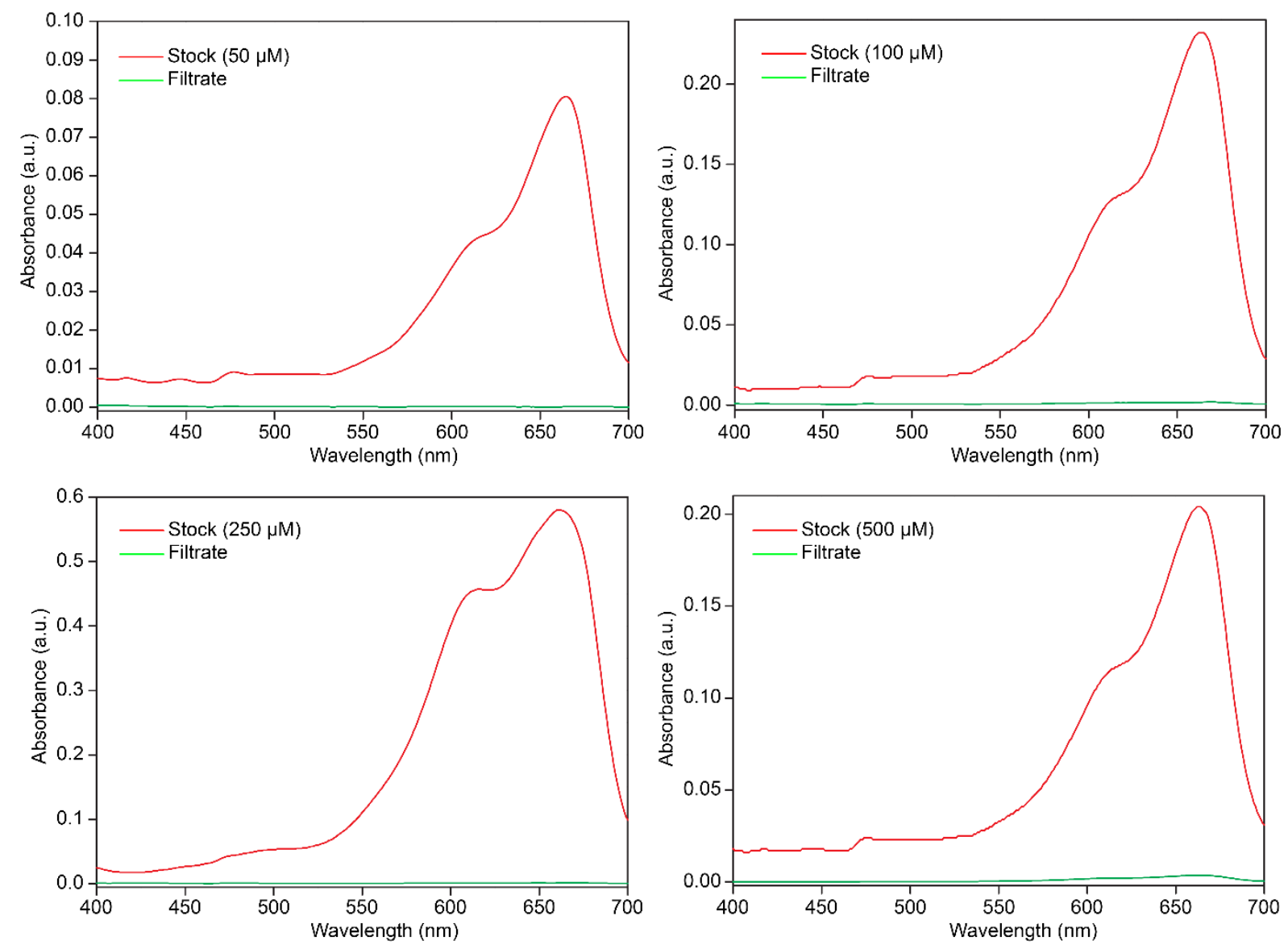

Figure S30 | Uptake capacity measurement. Pollutant removal efficiency of TpPa-2-foam at various pollutant concentrations has been represented here in. 


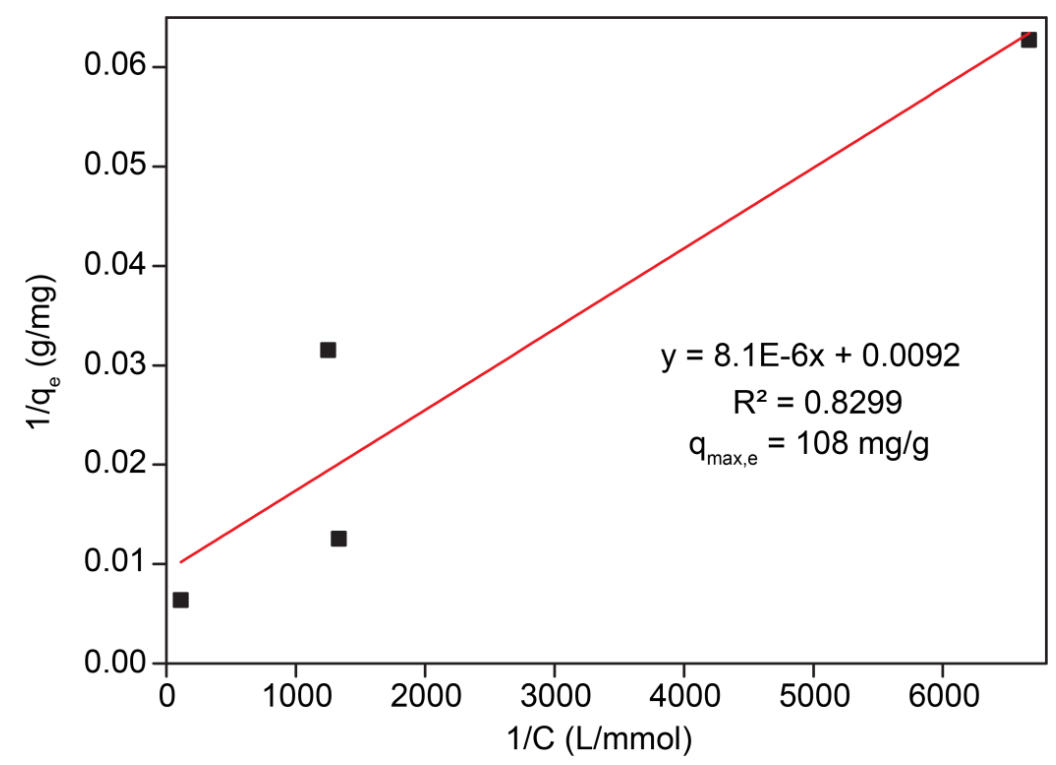

Figure S31 | Maximum pollutant uptake take capacity Maximum pollutant uptake capacity has been measured for TpPa-2-foam.
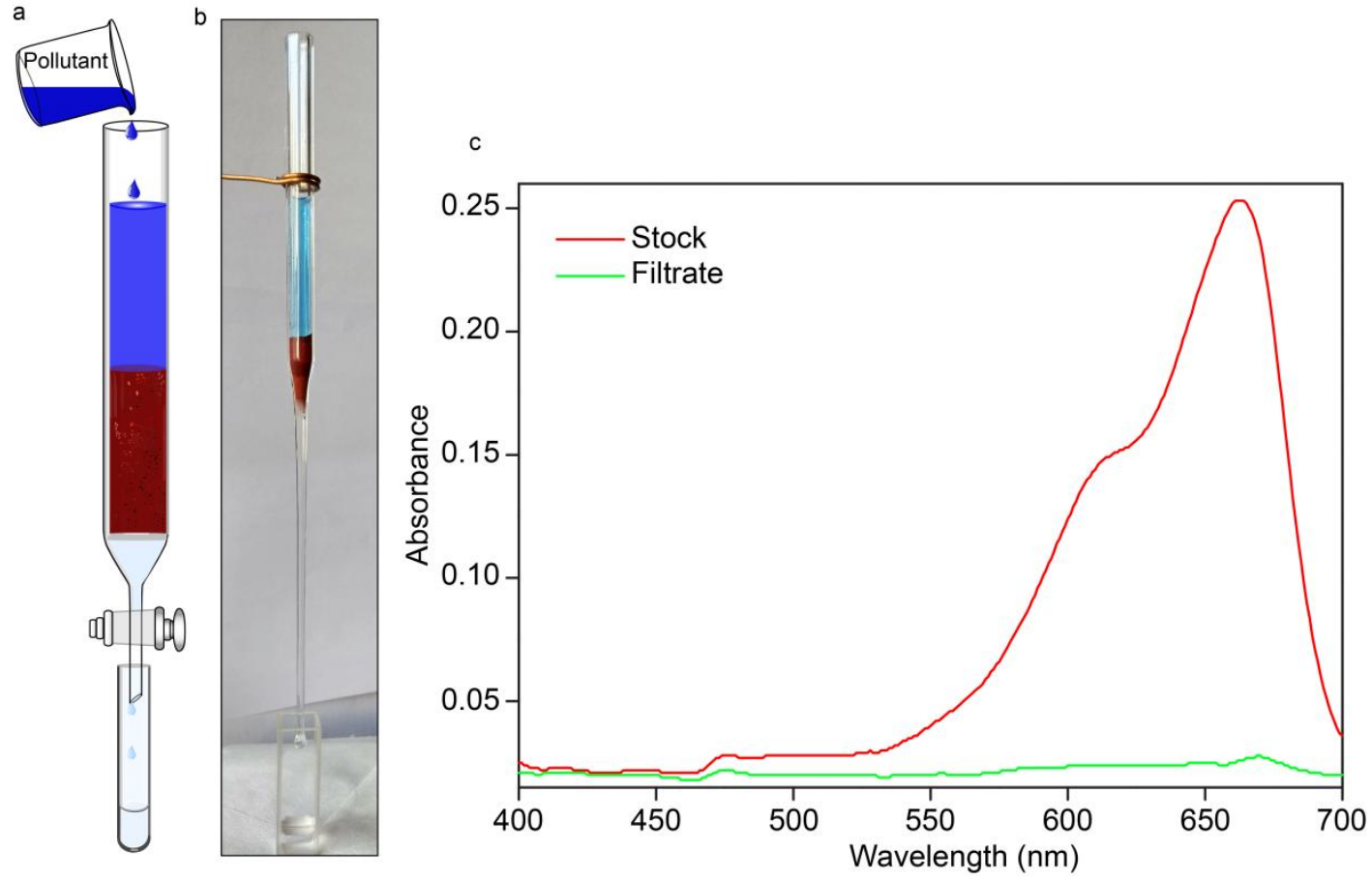

Figure S32 | Flow-through experiment. Pollutant (MB) removal efficiency has also been measure through a column bed of TpPa-2-foam. 

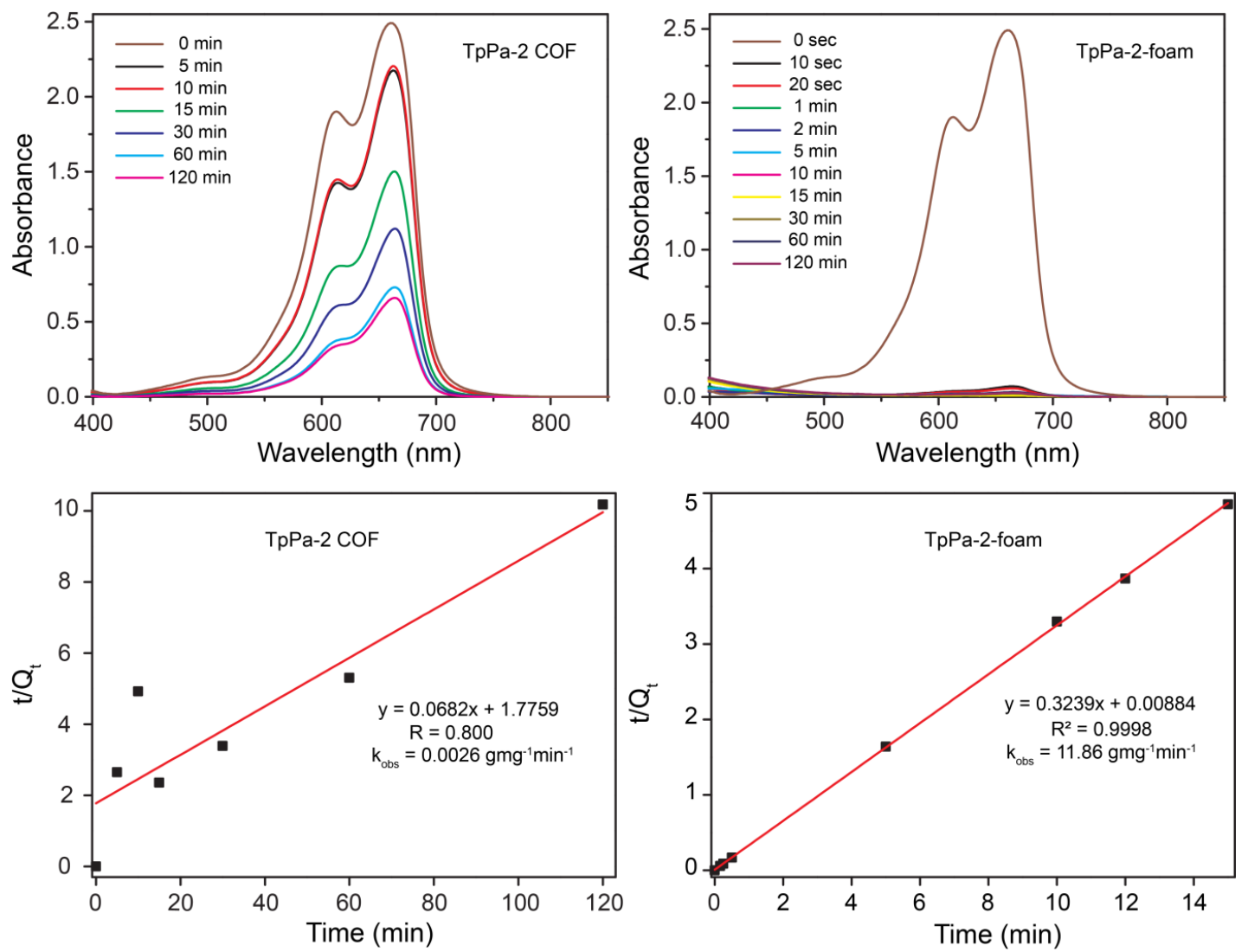

Figure S33 | Comparison of rate of adsorption. Kinetic adsorption has been performed for both TpPa-2 COF and COF-foam in order to make a comparison between their performances.

\section{Section S-11: Binding energy calculation}

TpPa-2-foam shows good removal efficiency for all the dyes irrespective of their molecular structures. The time-dependent confocal images also show that the pollutants are first adsorbed on the surface of the adsorbents and then diffuse through the extrinsic pores to the intrinsic ones. The surface adsorption, as well as the adsorption in the pores, could be attributed to the dispersive interactions (London forces) or supramolecular interaction of the pollutants with the adsorbents. Again, it is due to the hierarchical open porous structure of the foam, there is complete access of the molecular backbone of the framework which helps in better interactions. In the case of ionic dyes, there is a better dipole-dipole interaction that leads to very high removal efficiency. The $\mathrm{pH}$ of the medium generally contributes significantly during the adsorption by changing the chemical functionalities of the molecule 
due to protonation or deprotonation in acidic or basic medium. However, $\mathrm{TpPa}-2$ foam is highly stable in the entire $\mathrm{pH}$ range of $1-12$, and there is no chemical change in both the adsorbate (methylene blue) and adsorbents. Thus, changing the $\mathrm{pH}$ of the medium does not alter any dispersive interactions thereby unaffecting the adsorption properties of the foams. To validate the observation, we have also calculated the binding energy of the persistent organic pollutant, decabromodiphenyl ether (decaBDE), inside the defected layers of the TpPa-2-foam.All simulations were undertaken using the density-functional tight-binding (DFTB+) method including DFT-D3 dispersion. The calculations were carried out with the DFTB+ program package version 18.1 [DOI: 10.1021/jp070186p]. All atomic interactions were described with the 3ob-3-1 parameter set [Kubillus, M.; Kubař, T.; Gaus, M.; Řezáč, J.; Elstner, M. Parameterization of the DFTB3 Method for Br, Ca, Cl, F, I, K, and Na in Organic and Biological Systems (J. Chem. Theory Comput., 2015, 11, 332-342.). Thermodynamic minima of decaBDE@TpPa-2-foam were determined by the Kick3 stochastic structure search procedure [DOI: 10.1002/jcc.23420], allowing both ether bonds to be fully flexible. To model the foam, a three-layer COF was built and randomly terminated either at a node (aldehydeterminated) or linker ( $\mathrm{NH}_{2}$-terminated). $10 \AA$ of vacuum was added to all sides of the model COF-foam, and 100 starting geometries of a single decaBDE molecule and COF-foam model were generated and optimized (Figure S34 and Table S4). The calculation reveals that the binding energy of the pollutant on the trilayer of foam model varies in between $100-180$ $\mathrm{kJ} / \mathrm{mol}$.

Table S4 | Binding energy calculation for decabromodiphenyl ether inside the TpPa-2foam matrix.

\begin{tabular}{|c|c|c|c|c|}
\hline run & E (a.u.) & E_foam(a.u.) & E_pollutant(a.u.) & BE (kJ/mol) \\
\hline Kick0053 & $-1755,1639$ & $-1701,2883$ & $-53,8125461$ & $-165,52$ \\
\hline Kick0030 & $-1755,1525$ & $-1701,2671$ & $-53,81337456$ & $-189,11$ \\
\hline Kick0082 & $-1755,1481$ & $-1701,286$ & $-53,80846132$ & $-140,78$ \\
\hline Kick0032 & $-1755,148$ & $-1701,2728$ & $-53,81283701$ & $-163,61$ \\
\hline Kick0071 & $-1755,1415$ & $-1701,2666$ & $-53,81164467$ & $-165,93$ \\
\hline Kick0008 & $-1755,1407$ & $-1701,2693$ & $-53,81303287$ & $-153,26$ \\
\hline Kick0031 & $-1755,1399$ & $-1701,279$ & $-53,81362981$ & $-123,99$ \\
\hline Kick0057 & $-1755,1372$ & $-1701,2696$ & $-53,81360043$ & $-141,64$ \\
\hline Kick0045 & $-1755,135$ & $-1701,2731$ & $-53,81047976$ & $-135,02$ \\
\hline Kick0052 & $-1755,1313$ & $-1701,2777$ & $-53,81379695$ & $-104,44$ \\
\hline Kick0055 & $-1755,131$ & $-1701,2659$ & $-53,81282811$ & $-137,35$ \\
\hline Kick0046 & $-1755,1301$ & $-1701,2681$ & $-53,81180904$ & $-131,58$ \\
\hline Kick0021 & $-1755,1292$ & $-1701,2664$ & $-53,81322148$ & $-130,09$ \\
\hline Kick0050 & $-1755,1287$ & $-1701,2651$ & $-53,81156991$ & $-136,61$ \\
\hline Kick0036 & $-1755,1277$ & $-1701,2694$ & $-53,81403856$ & $-116,22$ \\
\hline
\end{tabular}




\begin{tabular}{|c|c|c|c|c|}
\hline Kick0041 & $-1755,1229$ & $-1701,2701$ & $-53,81322234$ & $-104,00$ \\
\hline Kick0029 & $-1755,1222$ & $-1701,2706$ & $-53,80927909$ & $-111,02$ \\
\hline Kick0020 & $-1755,1196$ & $-1701,2693$ & $-53,81060593$ & $-104,22$ \\
\hline Kick0001 & $-1755,1181$ & $-1701,2683$ & $-53,81138596$ & $-100,75$ \\
\hline Kick0093 & $-1755,1147$ & $-1701,2677$ & $-53,81333682$ & $-88,24$ \\
\hline Kick0047 & $-1755,1144$ & $-1701,2698$ & $-53,81413545$ & $-79,87$ \\
\hline Kick0037 & $-1755,113$ & $-1701,2671$ & $-53,81393321$ & $-83,85$ \\
\hline Kick0035 & $-1755,1128$ & $-1701,2693$ & $-53,81384411$ & $-78,06$ \\
\hline Kick0096 & $-1755,1127$ & $-1701,2707$ & $-53,81191865$ & $-78,98$ \\
\hline Kick0099 & $-1755,1116$ & $-1701,2696$ & $-53,81109252$ & $-80,97$ \\
\hline Kick0019 & $-1755,1112$ & $-1701,2714$ & $-53,8139016$ & $-68,02$ \\
\hline Kick0007 & $-1755,1109$ & $-1701,2714$ & $-53,81387911$ & $-67,32$ \\
\hline Kick0026 & $-1755,1106$ & $-1701,2738$ & $-53,81359265$ & $-61,01$ \\
\hline Kick0062 & $-1755,1093$ & $-1701,2636$ & $-53,81290422$ & $-86,21$ \\
\hline Kick0051 & $-1755,1087$ & $-1701,2727$ & $-53,81412002$ & $-57,67$ \\
\hline Kick0009 & $-1755,1086$ & $-1701,2731$ & $-53,81417938$ & $-56,05$ \\
\hline Kick0018 & $-1755,1082$ & $-1701,2755$ & $-53,81453909$ & $-47,68$ \\
\hline Kick0088 & $-1755,108$ & $-1701,2685$ & $-53,81425717$ & $-66,33$ \\
\hline Kick0006 & $-1755,1077$ & $-1701,2734$ & $-53,81435357$ & $-52,39$ \\
\hline Kick0025 & $-1755,1073$ & $-1701,273$ & $-53,81454694$ & $-51,86$ \\
\hline Kick0056 & $-1755,1057$ & $-1701,267$ & $-53,81418791$ & $-64,28$ \\
\hline Kick0064 & $-1755,1055$ & $-1701,2655$ & $-53,81379744$ & $-68,81$ \\
\hline Kick0058 & $-1755,1049$ & $-1701,2691$ & $-53,81380049$ & $-58,01$ \\
\hline Kick0039 & $-1755,1045$ & $-1701,2687$ & $-53,81436362$ & $-56,15$ \\
\hline Kick0000 & $-1755,1028$ & $-1701,2676$ & $-53,81443095$ & $-54,51$ \\
\hline Kick0090 & $-1755,1026$ & $-1701,2716$ & $-53,81424367$ & $-44,20$ \\
\hline Kick0004 & $-1755,1007$ & $-1701,272$ & $-53,81450858$ & $-37,10$ \\
\hline Kick0005 & $-1755,1004$ & $-1701,2695$ & $-53,8144052$ & $-43,40$ \\
\hline Kick0085 & $-1755,1003$ & $-1701,2687$ & $-53,81447148$ & $-44,97$ \\
\hline Kick0038 & $-1755,0998$ & $-1701,2702$ & $-53,81388583$ & $-41,48$ \\
\hline Kick0066 & $-1755,0983$ & $-1701,269$ & $-53,81435624$ & $-39,14$ \\
\hline Kick0076 & $-1755,0978$ & $-1701,27$ & $-53,81402931$ & $-35,98$ \\
\hline Kick0012 & $-1755,0963$ & $-1701,2672$ & $-53,81377012$ & $-40,30$ \\
\hline Kick0060 & $-1755,0955$ & $-1701,2697$ & $-53,814251$ & $-30,40$ \\
\hline Kick0077 & $-1755,0939$ & $-1701,2705$ & $-53,81443752$ & $-23,55$ \\
\hline Kick0084 & $-1755,0938$ & $-1701,2667$ & $-53,8142794$ & $-33,55$ \\
\hline Kick0063 & $-1755,0923$ & $-1701,2691$ & $-53,81458468$ & $-22,59$ \\
\hline Kick0028 & $-1755,0915$ & $-1701,2703$ & $-53,81448972$ & $-17,73$ \\
\hline Kick0042 & $-1755,0914$ & $-1701,2685$ & $-53,81419024$ & $-22,86$ \\
\hline Kick0078 & $-1755,0909$ & $-1701,2718$ & $-53,81462191$ & $-11,73$ \\
\hline Kick0087 & $-1755,0903$ & $-1701,2698$ & $-53,81458455$ & $-15,37$ \\
\hline Kick0043 & $-1755,0902$ & $-1701,2698$ & $-53,81460464$ & $-15,09$ \\
\hline Kick0016 & $-1755,0899$ & $-1701,2741$ & $-53,8146408$ & $-3,10$ \\
\hline Kick0097 & $-1755,0897$ & $-1701,271$ & $-53,81462386$ & $-10,84$ \\
\hline Kick0017 & $-1755,0895$ & $-1701,2693$ & $-53,81458126$ & $-14,70$ \\
\hline Kick0094 & $-1755,0893$ & $-1701,27$ & $-53,81454833$ & $-12,61$ \\
\hline Kick0081 & $-1755,0887$ & $-1701,2713$ & $-53,81461738$ & $-7,24$ \\
\hline Kick0069 & $-1755,0884$ & $-1701,2715$ & $-53,81462371$ & $-6,08$ \\
\hline
\end{tabular}




\begin{tabular}{|c|c|c|c|c|}
\hline Kick0070 & $-1755,0876$ & $-1701,2697$ & $-53,81457803$ & $-8,77$ \\
\hline Kick0059 & $-1755,0874$ & $-1701,272$ & $-53,81462959$ & $-2,14$ \\
\hline Kick0091 & $-1755,0873$ & $-1701,2718$ & $-53,81462742$ & $-2,55$ \\
\hline Kick0079 & $-1755,0871$ & $-1701,2696$ & $-53,81456761$ & $-7,69$ \\
\hline Kick0034 & $-1755,0871$ & $-1701,272$ & $-53,81464963$ & $-1,09$ \\
\hline Kick0024 & $-1755,0868$ & $-1701,2714$ & $-53,81465178$ & $-2,22$ \\
\hline Kick0095 & $-1755,0868$ & $-1701,2701$ & $-53,81457562$ & $-5,49$ \\
\hline Kick0003 & $-1755,0866$ & $-1701,2713$ & $-53,81465081$ & $-1,58$ \\
\hline Kick0010 & $-1755,0866$ & $-1701,2712$ & $-53,81464215$ & $-1,86$ \\
\hline Kick0027 & $-1755,0865$ & $-1701,2713$ & $-53,81464698$ & $-1,46$ \\
\hline Kick0073 & $-1755,0863$ & $-1701,2702$ & $-53,81463854$ & $-3,93$ \\
\hline Kick0033 & $-1755,0862$ & $-1701,2711$ & $-53,81463657$ & $-1,07$ \\
\hline Kick0068 & $-1755,0861$ & $-1701,2711$ & $-53,81464125$ & $-0,70$ \\
\hline Kick0014 & $-1755,086$ & $-1701,2711$ & $-53,8146507$ & $-0,68$ \\
\hline Kick0061 & $-1755,086$ & $-1701,2707$ & $-53,81463943$ & $-1,68$ \\
\hline Kick0089 & $-1755,0859$ & $-1701,2703$ & $-53,81463001$ & $-2,56$ \\
\hline Kick0049 & $-1755,0858$ & $-1701,2702$ & $-53,81463107$ & $-2,60$ \\
\hline Kick0015 & $-1755,0858$ & $-1701,2709$ & $-53,81460075$ & $-0,88$ \\
\hline Kick0013 & $-1755,0857$ & $-1701,2698$ & $-53,81458854$ & $-3,64$ \\
\hline Kick0072 & $-1755,0857$ & $-1701,2704$ & $-53,81462086$ & $-1,65$ \\
\hline Kick0080 & $-1755,0856$ & $-1701,2691$ & $-53,81461549$ & $-4,94$ \\
\hline Kick0074 & $-1755,0855$ & $-1701,2704$ & $-53,81461147$ & $-1,40$ \\
\hline Kick0083 & $-1755,0855$ & $-1701,27$ & $-53,81459518$ & $-2,38$ \\
\hline Kick0086 & $-1755,0854$ & $-1701,2706$ & $-53,81461382$ & $-0,62$ \\
\hline Kick0075 & $-1755,0854$ & $-1701,2706$ & $-53,81462149$ & $-0,45$ \\
\hline Kick0054 & $-1755,0854$ & $-1701,2696$ & $-53,81463131$ & $-3,13$ \\
\hline Kick0044 & $-1755,0854$ & $-1701,2701$ & $-53,81460215$ & $-1,66$ \\
\hline Kick0011 & $-1755,0854$ & $-1701,2702$ & $-53,81462044$ & $-1,33$ \\
\hline Kick0048 & $-1755,0854$ & $-1701,2702$ & $-53,81461728$ & $-1,50$ \\
\hline Kick0023 & $-1755,0853$ & $-1701,2698$ & $-53,81459232$ & $-2,55$ \\
\hline Kick0065 & $-1755,0853$ & $-1701,2703$ & $-53,8146298$ & $-0,91$ \\
\hline Kick0067 & $-1755,0852$ & $-1701,2702$ & $-53,8146167$ & $-1,00$ \\
\hline Kick0002 & $-1755,0852$ & $-1701,2703$ & $-53,81462176$ & $-0,62$ \\
\hline Kick0098 & $-1755,0849$ & $-1701,2699$ & $-53,81461433$ & $-1,25$ \\
\hline Kick0022 & $-1755,0849$ & $-1701,2696$ & $-53,81457177$ & $-1,79$ \\
\hline Kick0092 & $-1755,0748$ & $-1701,2598$ & $-53,81460731$ & $-0,96$ \\
\hline
\end{tabular}




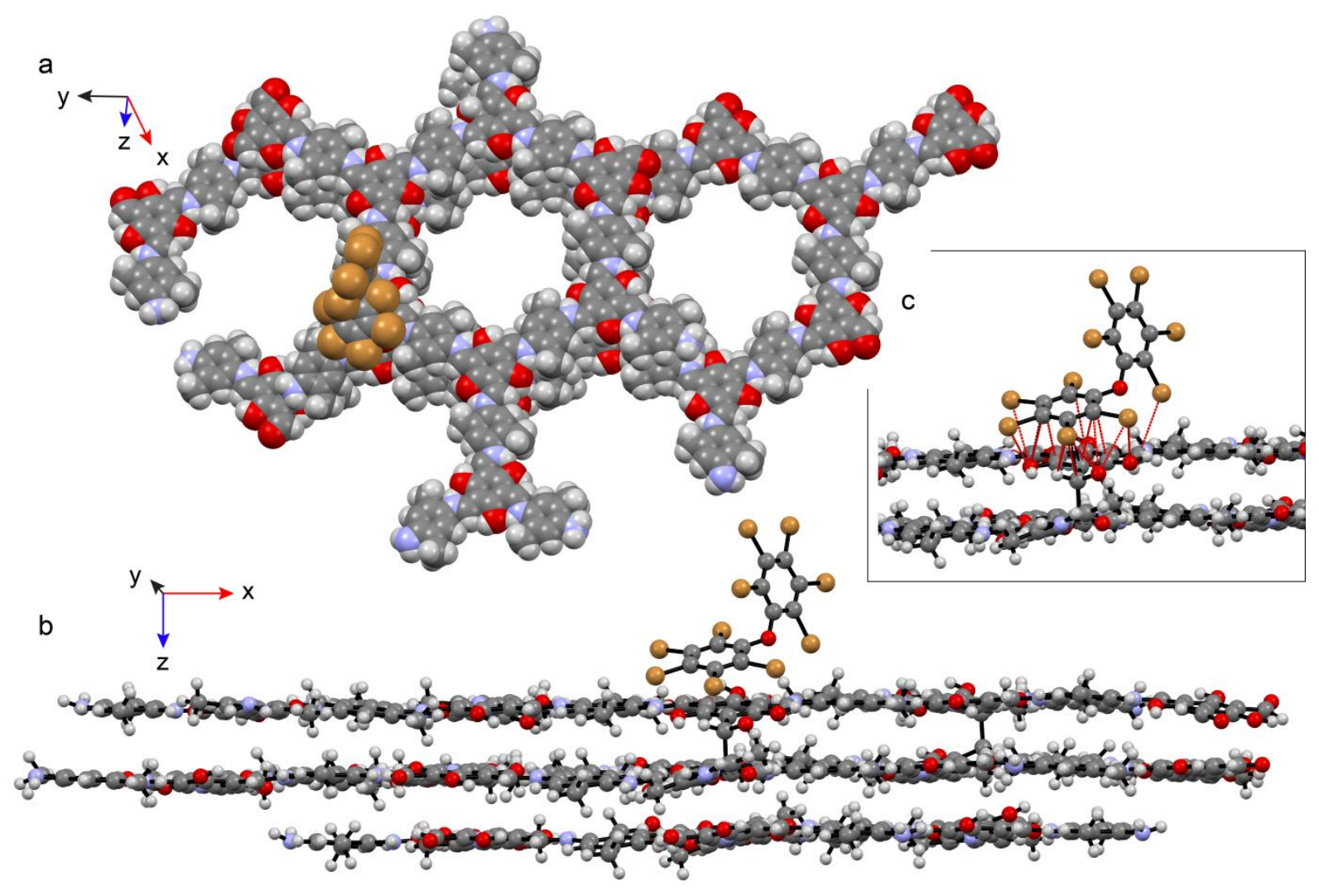

Figure S34 | The energy optimized structure of decaBDE inside the foam-matrix shows the possible bindings with the framework.

\section{Section S-12: Numerical simulation studies}

Pore characteristics and fluid flow properties of COF's were computed from their respective 3D image data sets using PoroDict and FlowDict (GeoDict 2018, Math2Market GmBH, Germany) software packages, respectively., $2 \mathrm{D}$ images were filtered to remove noise and segmented after manual threshold selection based on local minima from the grayscale histogram. Resultant 3D reconstructed model is subjected to pore-size distribution algorithm using PoroDict software package, where pore radius is determined by fitting spheres into the pore volume. Mean flow velocity in all three axis of the 3D model is predicted for a given pressure drop using FlowDict software package. In FlowDict's post-processing step, the relationship between the predicted pressure drop, fluid viscosity and media thickness expressed in Darcy's law is used to compute and output the material permeability. 

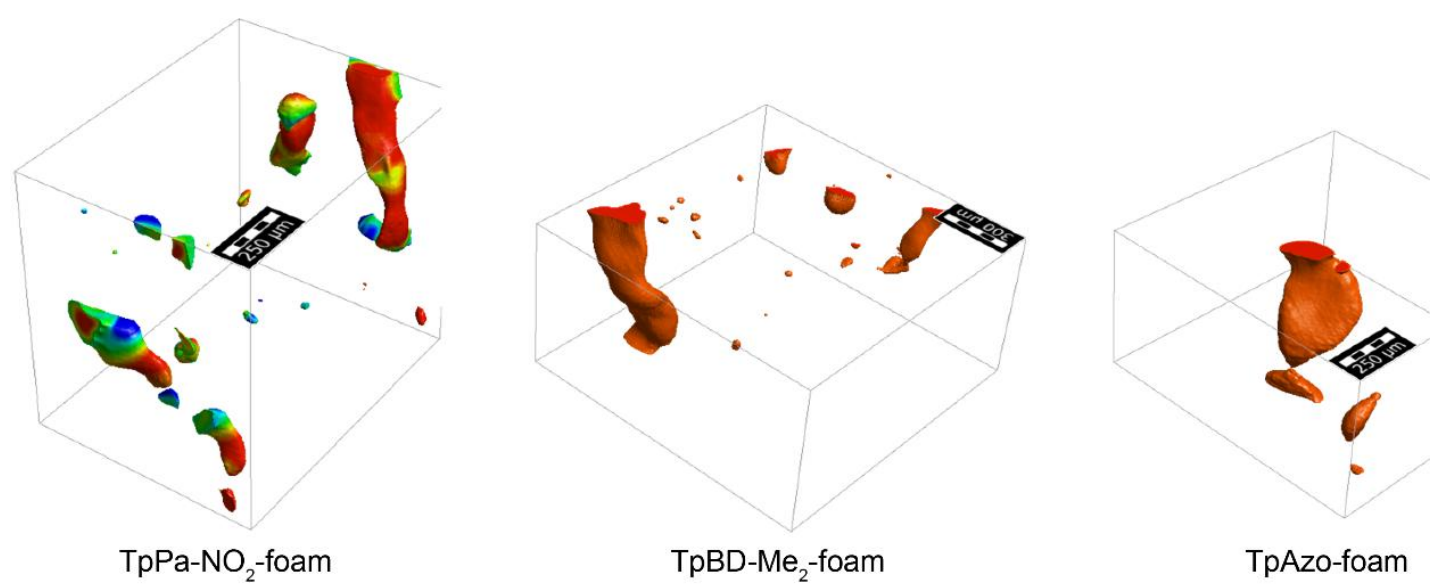

Figure S35 | Flow velocity measurement. The flow velocity simulation study has been performed for all the COF-foams. The average flow velocity has been calculated from the simulation.

\section{Section S-13: References}

1. Chong, J. H.; Sauer, M.; Patrick, B. O.; MacLachlan, M. J. Org. Lett. 2003, 5, 38233826.

2. Shinde, D. B.; Aiyappa, H. B.; Bhadra, M.; Biswal, B. P.; Wadge, P.; Kandambeth, S.; Garai, B.; Kundu, T.; Kurungot, S.; Banerjee, R. J. Mater. Chem. A 2016, 4, 26822690.

3. Ohser, J. \& Mücklich, F. Statistical Analysis of Microstructures in Materials Science 115 (Wiley and Sons, 2000).

4. Ohser, J. \& Schladitz, K. 3D Images of Materials Structures; Processing and Analysis 281 (Wiley-VCH Verlang GmbH, 2009). 